\title{
Development of Unified High-Fidelity Flight Dynamic Modeling Technique for Unmanned Compound Aircraft
}

\author{
Do hyeon Lee, ${ }^{1}$ Chang-joo Kim $\mathbb{D}^{2},{ }^{2}$ and Seong han Lee ${ }^{3}$ \\ ${ }^{1}$ Turret Technology Team, Hanwha Defense, Seongnam-si 13488, Republic of Korea \\ ${ }^{2}$ Konkuk University, Seoul 05029, Republic of Korea \\ ${ }^{3}$ Avionics System Team, Hanwha System, Seongnam-si 13524, Republic of Korea \\ Correspondence should be addressed to Chang-joo Kim; cjkim@konkuk.ac.kr
}

Received 23 February 2021; Revised 14 April 2021; Accepted 19 April 2021; Published 4 May 2021

Academic Editor: Jacopo Serafini

Copyright @ 2021 Do hyeon Lee et al. This is an open access article distributed under the Creative Commons Attribution License, which permits unrestricted use, distribution, and reproduction in any medium, provided the original work is properly cited.

\begin{abstract}
This study presents the unified high-fidelity flight dynamic modeling technique for compound aircraft. The existing flight dynamic modeling technique is absolutely depended on the experimental data measured by wind tunnel. It means that the existing flight dynamic model cannot be used for analyzing a new configuration aircraft. The flight dynamic modeling has to be implemented when a performance analysis has to be performed for new type aircraft. This technique is not effective for analyzing the performance of the new configuration aircraft because the shapes of compound aircraft are very various. The unified highfidelity flight dynamic modeling technique is developed in this study to overcome the limitation of the existing modeling technique. First, the unified rotor and wing models are developed to calculate the aerodynamic forces generated by rotors and wings. The revolutions per minute (RPM) and pitch change with rotation direction are addressed by rotor models. The unified wing model calculates the induced velocity by using the vortex lattice method (VLM) and the Biot-Savart law. The aerodynamic forces and moments for wings and rotors are computed by strip theory in each model. Second, the performance analysis such as propeller performance and trim for compound aircraft is implemented to check the accuracy between the proposed modeling technique and the helicopter trim, linearization, and simulation (HETLAS) program which is validated. It is judged that this study raises the efficiency of aircraft performance analysis and the airworthiness evaluation.
\end{abstract}

\section{Introduction}

Unmanned aerial vehicles (UAVs) are used for various purposes in military and civilian applications owing to their enormous advantages such as wide range of operational missions and flight altitudes [1]. It is necessary to develop a suitable configuration for the operational purpose and accurately analyze the flight performance as the demand for UAVs in the aviation industry. Today, the various maneuvers such as hovering, low-speed flight, and high-speed flight are simultaneously required to UAVs. However, the fixed-wing aircrafts cannot easily perform hovering and low-speed flight mission. Moreover, the rotorcrafts cannot implement the high-speed flight mission due to the problem of compressibility and flow separation in the rotor. For this reason, many aircraft manu- facturers and researchers have proposed the compound UAVs. They conducted performance analysis and verification for compound UAVs. The most famous configurations are the vertical/short takeoff and landing (V/STOL) wheel [2]. Figure 1 shows the V/STOL aircraft and propulsion concepts.

Basset et al. [3] tried to define the five categories of rotorcraft shapes as shown in Figure 2. The categories mean that all of compound aircraft can be represented by combinations of rotating components such as rotors, propellers, and fixed components such as wings and engines. Thus, the flight dynamic modeling techniques are the most important section when the designer is developing the compound aircraft.

The traditional modeling techniques are considerably depending on the wind tunnel data $[4,5]$. This method is 


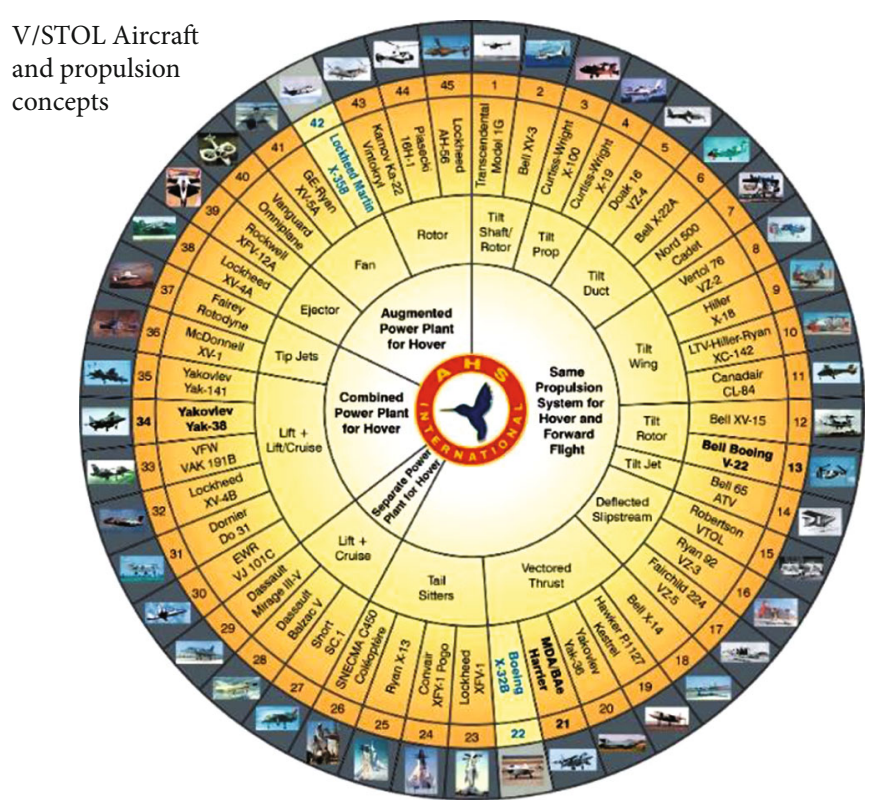

Figure 1: V/STOL wheel [2].

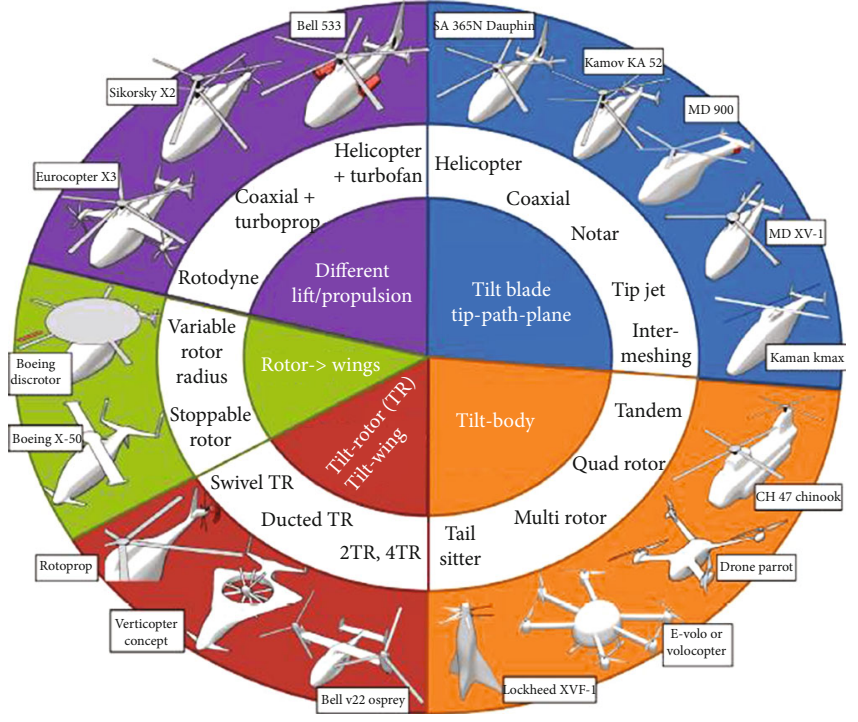

FIGURE 2: Five categories of rotorcrafts [3].

not efficiency because the experimental using wind tunnel has to be perform for each aircraft. It is the limitation to develop the aircraft system since the development cost and period are highly raised. Also, it is difficult to modify the flight dynamic model (FDM) according to the purpose of analysis or to add functions since the most FDM is distributed as commercial program [6]. The flight dynamic modeling techniques have been developed by several researchers to overcome this disadvantage. Kim et al. [7] studied the aerodynamic and inertial modeling of propeller. Cook et al. [4] and Etkins et al. [5] researched the flight dynamic principle. (1) Cook MV and Etkins used the traditional modeling techniques using wind tunnel data. Leishman et al. [8] tried to develop the mathematical helicopter aerodynamic model. Chaffin et al. [9] defined the guide of the use for pressure disk rotor model. Taamallah et al. [10] studied the flight dynamic modeling for a smallscale helicopter UAV. Howlett et al. [11] developed the black hawk engineering simulation program using mathematical model. Pearson et al. [12] researched the aerodynamic characteristics of tapered wings. Talbot et al. [13] studied the mathematical model for single main rotor helicopter. The researchers in Ref [8-13] had tried to develop the mathematical modeling technique for helicopter. However, (2) these researches can be used for certain aircraft. If the configurations of aircraft are changed, the flight dynamic modeling procedure has to be newly started. This is the limitation for developing compound aircraft because it is difficult to develop the flight dynamic model for each compound aircraft. It raises the development cost and period. So, most companies designing the aircraft system cannot try to develop the compound aircraft. (3) Therefore, the flight dynamic modeling technique has to be developed to easily express the characteristics of all type compound aircraft.

The contribution of this studied is the development of unified flight dynamic modeling technique to overcome the disadvantages of existing modeling techniques and to easily improve the fidelity of FDM while responding to changes in aircraft geometry. Especially, a unified rotor and wing models are developed to selectively calculate the aerodynamic forces of the rotors with wings and to analyze the flight performance of any aircraft without modifying the mathematical model. This technique can easily modify and add the rotor or wing models according to the analysis purpose.

The rest of this paper is organized as follows. Section 2 introduces the unified aerodynamic component modeling technique, and Section 3 deals with the application to validate the accuracy of proposed modeling method by comparing with the HETLAS program. Section 4 is the conclusion of this research. 


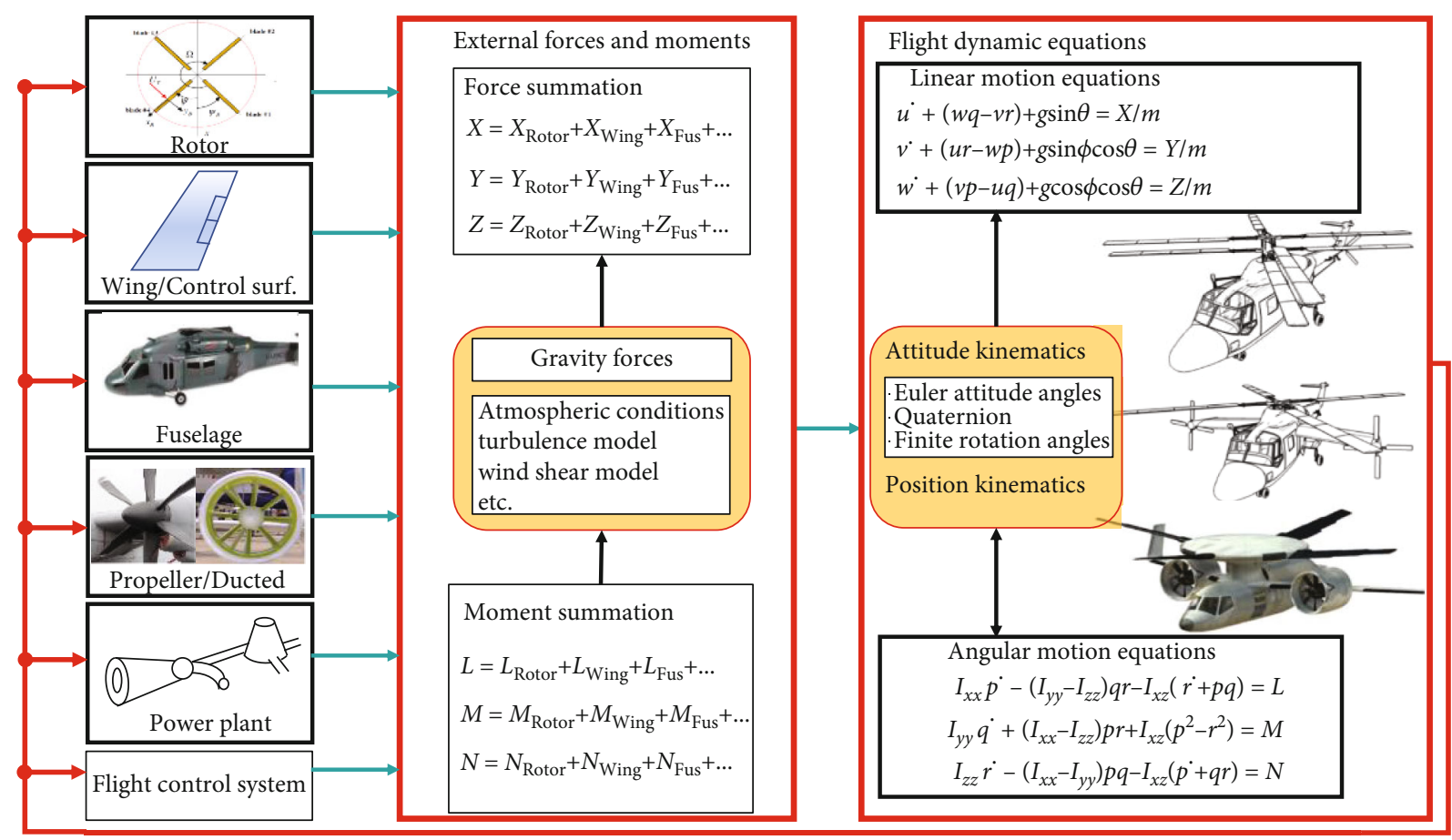

FIgURE 3: Component-based modeling technique.

TABLE 1: Classification of various rotors.

\begin{tabular}{|c|c|c|c|}
\hline Rotor type & Mounting position and orientation & Available dynamics & Control mechanism \\
\hline $\begin{array}{l}\text { Conventional main } \\
\text { rotor }\end{array}$ & $\begin{array}{c}\text {-Top/center of fuselage } \\
\text {-Vertical (reference) with small FWD } \\
\text { tilt angle }\end{array}$ & Flap/lag/RPM & Collective and 2 cyclic pitches \\
\hline $\begin{array}{l}\text { Conventional tail } \\
\text { rotor }\end{array}$ & $\begin{array}{c}\text {-Rear fuselage } \\
- \pm 90 \text { deg sideward tilt with small cant } \\
\text { angle }\end{array}$ & $\begin{array}{l}\text { Flap/RPM (MR } \\
\text { dependent) }\end{array}$ & Collective (pedal) \\
\hline Teetering main rotor & $\begin{array}{l}\text {-Top/center of fuselage } \\
\text {-Vertical with small FWD tilt angle }\end{array}$ & Flap & Collective and 2 cyclic pitches \\
\hline Teetering tail rotor & $\begin{array}{c}- \text { Rear fuselage } \\
- \pm 90 \text { deg sideward tilt with small cant } \\
\text { angle }\end{array}$ & Flap & Collective (pedal) \\
\hline $\mathrm{ABC}$ rotor (coaxial) & $\begin{array}{l}\text {-Top/center of fuselage } \\
\text {-Vertical with small FWD tilt angle }\end{array}$ & No & $\begin{array}{c}\text { Collective and } 2 \text { cyclic pitches with differential } \\
\text { collective }\end{array}$ \\
\hline Propeller & $\begin{array}{l}\text {-Front (tractor) or rear (pusher) of } \\
\text { fuselage } \\
- \pm 90 \text { deg FWD tilt angle }\end{array}$ & No & Collective or RPM \\
\hline Ducted fan & -Design dependent & No & Collective (thrust vectoring) \\
\hline
\end{tabular}

\section{Unified Aerodynamic Component Modeling Technique}

This section describes the unified aerodynamic component modeling techniques for FDM. The traditional flight dynamic modeling techniques have used the wind tunnel test data as mentioned in introduction. Its method allows to build the high-fidelity FDM. However, if the configurations of aircraft are changed, the FDM developed by the traditional modeling technique cannot be used for new aircraft. Thus, this paper uses the unified component-based modeling technique as shown in Figure 3. This technique builds each component model such as rotor, wing, fuselage, propeller, and duct after modeling the aerodynamic forces and moments. The FDM of all type configurations for compound aircraft can be easily developed, and the flight dynamics analysis is rapidly implemented. The main component model generating aerodynamic forces and moments is the rotors and wings. Therefore, the aerodynamic modeling techniques of rotors and wings are developed in this section. 


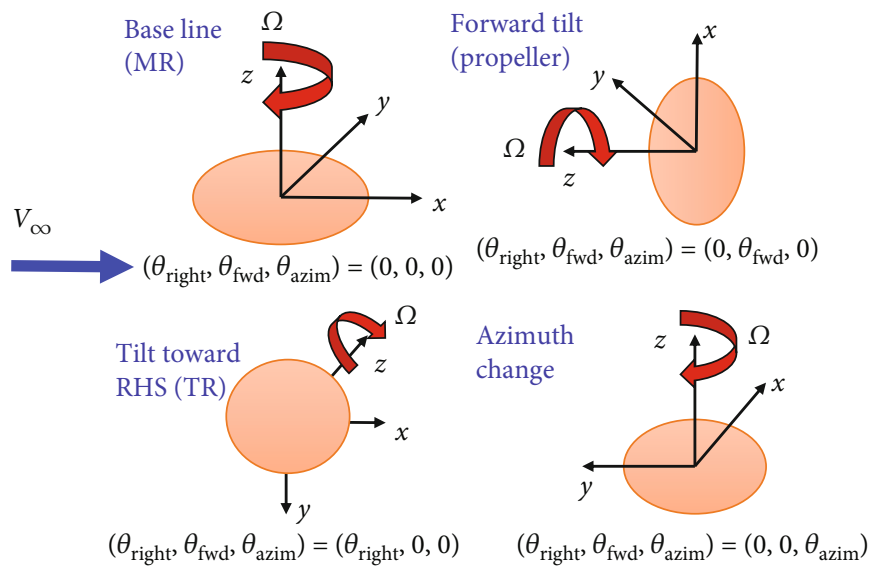

Figure 4: Handling rotor orientation for the unified rotor model.

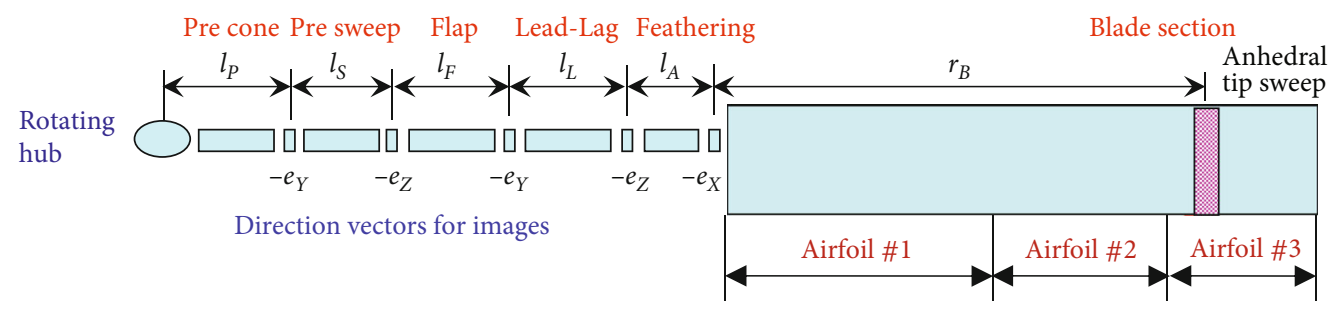

Figure 5: General information for rotor model [15].

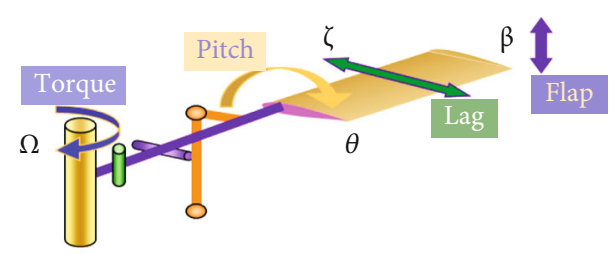

FIGURE 6: General information for rotor dynamics with rigid blade.

TABLE 2: Variables for rotor dynamics (variables for blade motions).

\begin{tabular}{lc}
\hline Variable & Description \\
\hline$l_{P}$ & Precone offset distance from hub frame \\
$l_{S}$ & Presweep offset distance from precone \\
$l_{F}$ & Flap offset distance from presweep \\
$l_{L}$ & Lead-lag offset distance from flap hinge \\
$l_{A}$ & Feathering offset distance from lead-lag hinge \\
$e_{X}$ & Unit vector of $X$-direction \\
$e_{Y}$ & Unit vector of $Y$-direction \\
$e_{Z}$ & Unit vector of $Z$-direction \\
$r_{B}$ & Distance between feathering hinge to blade section \\
$\Omega$ & Rotational speed of rotor \\
$\theta$ & Feathering angle \\
$\zeta$ & Lagging angle \\
$\beta$ & Flapping angle \\
\hline
\end{tabular}

2.1. Unified Rotor Modeling. The rotors can be classified depending on whether they produce the thrust or propulsive forces. The mathematical aerodynamic model of the rotors can be expressed by the blade element method (BEM). However, there are differences between a rotor and a propeller in terms of the mounting position, orientation, and rotor dynamics such as flapping motion. Therefore, various shapes of the rotor and propeller are classified according to the mounting position, orientation, rotor dynamics, and control mechanism as presented in Table 1.

Table 1 presents some standardized rotor types. The reference position and direction should be selected to indicate the mounting position and direction of the rotor using the unified rotor model. The aerodynamic forces and moments can be expressed on the body coordinate because all the forces and moments of the aircraft act on the center of gravity (CG). Therefore, the mounting position of the rotor is expressed using the relative distance vector from the CG of the aircraft.

2.1.1. Mounting Position and Orientation. The main rotor of a conventional helicopter is located at the top center of the aircraft and mounted perpendicular to the fuselage or with small forward tilt angles. The tail rotor is generally located on the rear of the aircraft and mounted vertically on the lateral axis. The propeller is mounted on the front (tractor type) or the rear (pusher type) perpendicular to the axis of the fuselage. Here, the azimuth changes for main rotor (MR), propeller, and tail rotor (TR) are expressed as shown in Figure 4. 

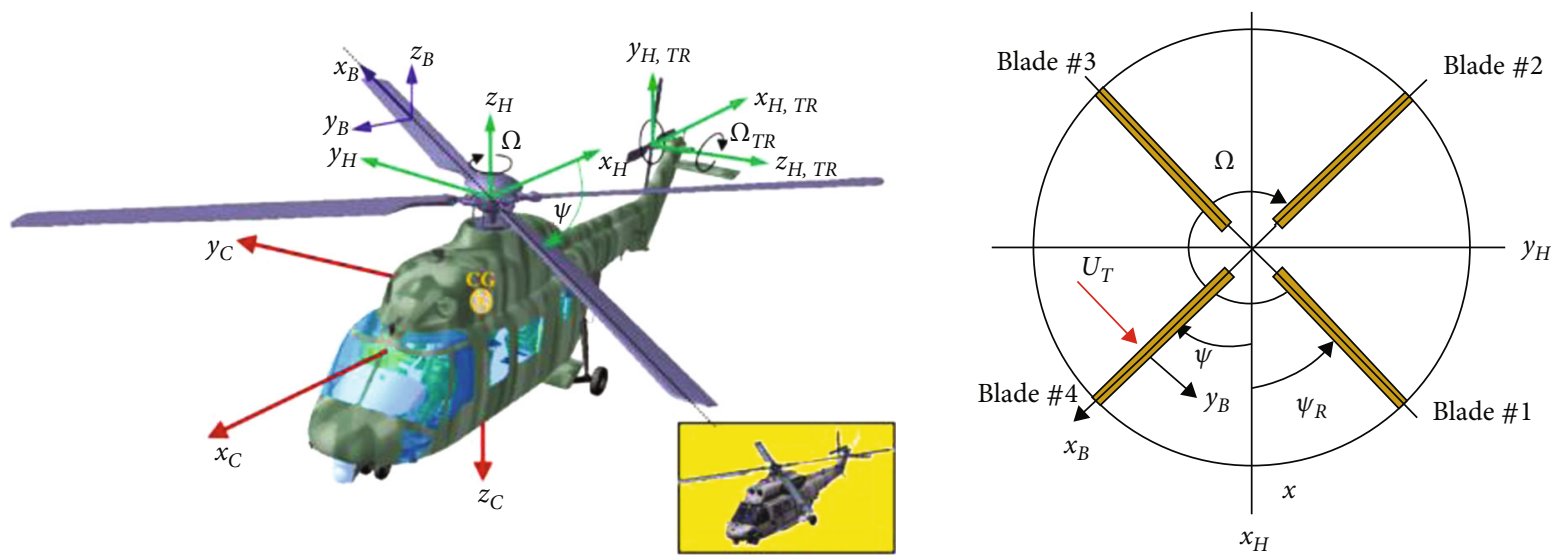

FIgURE 7: The coordinate system for the helicopter and blade elements.

TABLE 3: Variables for rotor dynamics.

\begin{tabular}{l} 
Variable \\
\hline$m_{b}$ \\
$g_{C}^{C I}, \overline{\mathbf{d}}_{F}$ \\
$\mathbf{d}_{L}, \overline{\mathbf{d}}_{L}$ \\
$\mathbf{d}_{R}, \overline{\mathbf{d}}_{R}$ \\
$C_{\dot{\beta}}, C_{\dot{\zeta}}$ \\
$K_{\beta}, K_{\zeta}$
\end{tabular}

FIGURE 8: General information for generating aerodynamic forces with induced drag.

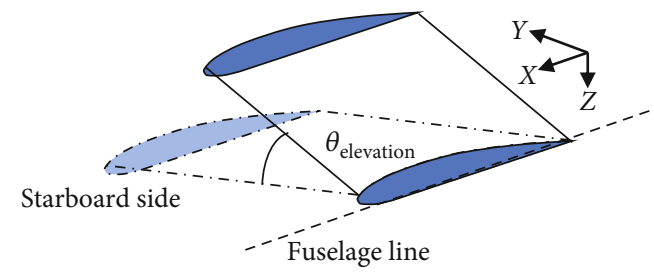

FIGURE 9: Wing orientation.

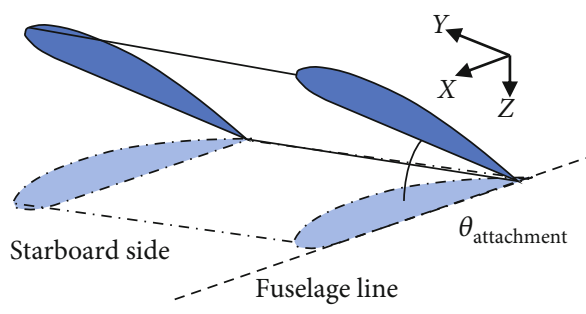

Figure 10: Wing attachment.

The mounting angles are defined to represent the mounting orientation, namely, the starboard side tilt angle $\theta_{\text {Right }}$, which rotates in the CCW direction on the $x$-axis, the forward tilt angle $\theta_{F W D}$, which rotates in the CCW (counter clockwise) direction on the $y$-axis, and finally, the azimuth rotation angle $\theta_{A Z I M}$, which rotates in the CCW direction on the $z$-axis. The passive transformation (alias transformation) of the coordinate system applied to the individual rotor is defined as given in Equation (1) for each axis. The matrices $\mathbf{L}_{1}, \mathbf{L}_{2}, \mathbf{L}_{3}$ are the transformation matrices around $x$-, $y$-, and $z$-axis, respectively. 


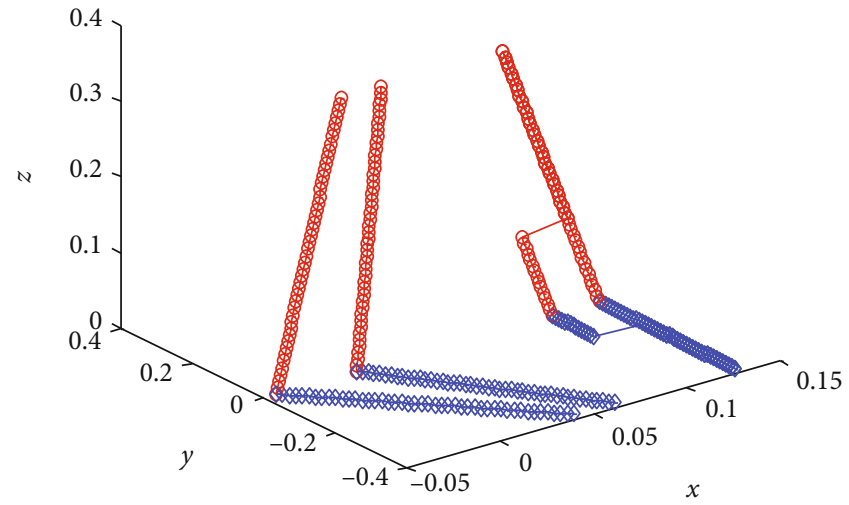

FIgURE 11: Wing orientation of the $\theta_{\text {Elev }}$ in the $X-Y-Z$ coordinate.

$$
\begin{aligned}
& \mathbf{L}_{1}(x)=\left(\begin{array}{ccc}
1 & 0 & 0 \\
0 & \cos x & \sin x \\
0 & -\sin x & \cos x
\end{array}\right), \\
& \mathbf{L}_{2}(y)=\left(\begin{array}{ccc}
\cos y & 0 & -\sin y \\
0 & 1 & 0 \\
\sin y & 0 & \cos y
\end{array}\right), \\
& \mathbf{L}_{3}(z)=\left(\begin{array}{ccc}
\cos z & \sin z & 0 \\
-\sin z & \cos z & 0 \\
0 & 0 & 1
\end{array}\right) .
\end{aligned}
$$

The reference for the mounting orientation is the orientation of the conventional main rotor. The coordinate transformation matrix from CG to the rotor hub $\mathrm{L}_{\mathrm{HC}}$ is defined as given in Equation (2).

$$
\mathbf{L}_{\mathrm{HC}}=\mathbf{L}_{1}\left(\theta_{\text {Right }}\right) \mathbf{L}_{2}\left(\pi-\theta_{\mathrm{FWD}}\right) \mathbf{L}_{3}\left(\theta_{\mathrm{AZIM}}\right) .
$$

Various rotor orientations can be expressed using this transformation matrix. For instance, the conventional main rotor is represented as $\left(\theta_{\text {Right }}, \theta_{\mathrm{FWD}}, \theta_{\mathrm{AZIM}}\right)=(0,0,0)$, whereas the pusher propeller and tail rotor mounted on the starboard side are expressed as $\left(\theta_{\text {Right }}, \theta_{\text {FWD }}, \theta_{\text {AZIM }}\right)=(-\pi / 2$ $, 0,0)$ and $\left(\theta_{\text {Right }}, \theta_{\text {FWD }}, \theta_{\text {AZIM }}\right)=(0, \pi / 2,0)$ as shown in Figure 4 , respectively.

2.1.2. Rotor Dynamics. The rotor and the propeller are separated based on the rotor dynamics. The rotor dynamics is not applied when the propeller and ABC (advanced blade concept) rotor developed by Sikorsky are used [14]. Figures 5 and 6 show the general information for rotor dynamics. The unified rotor dynamics can select the rotor dynamics for each rotor. Table 2 is the descriptions for variables of rotor dynamics. The aerodynamic forces and moments of the blade elements are calculated using BEM. The aerodynamic coefficients corresponding to the angle of attack (AOA) and Mach number of the blade elements are estimated from the nonlinear aerodynamic coefficient table con-

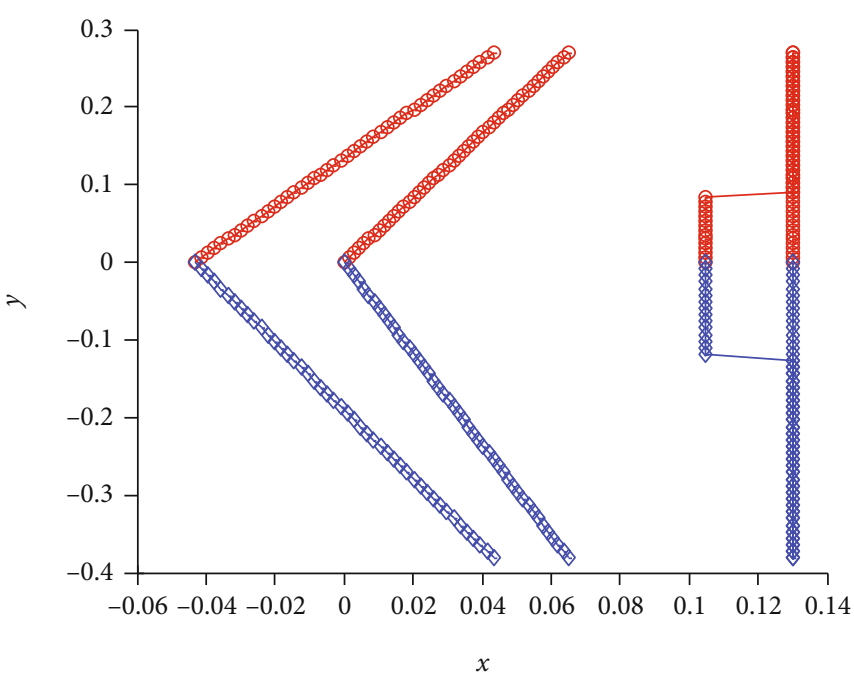

FIgURE 12: Wing orientation of the $\theta_{\text {Elev }}$ in the $X-Y$ coordinate.

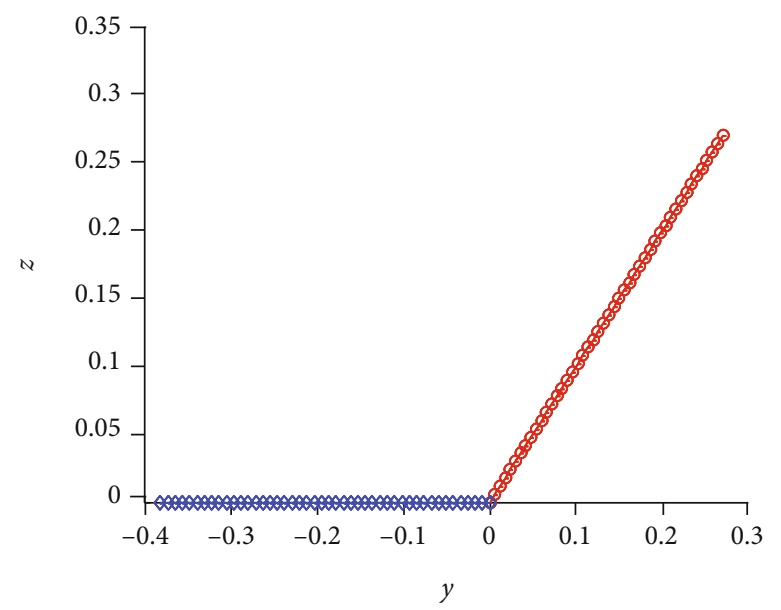

FIgURE 13: Wing orientation of the $\theta_{\text {Elev }}$ in the $Y-Z$ coordinate.

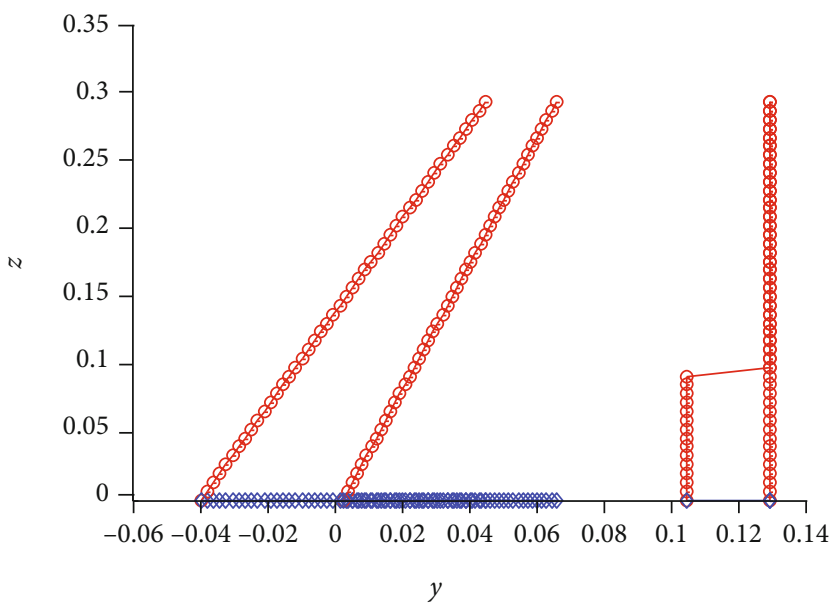

FIgURE 14: Wing orientation of the $\theta_{\text {Elev }}$ in the $X-Z$ coordinate. 


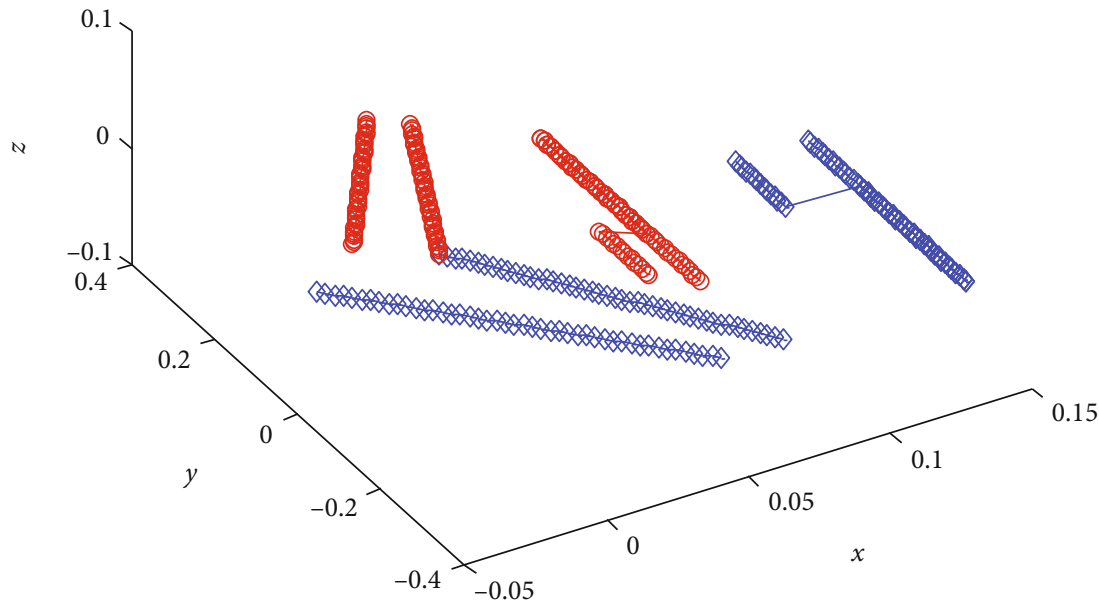

Figure 15: Wing orientation of the $\theta_{\text {Attach }}$ in the $X-Y-Z$ coordinate.

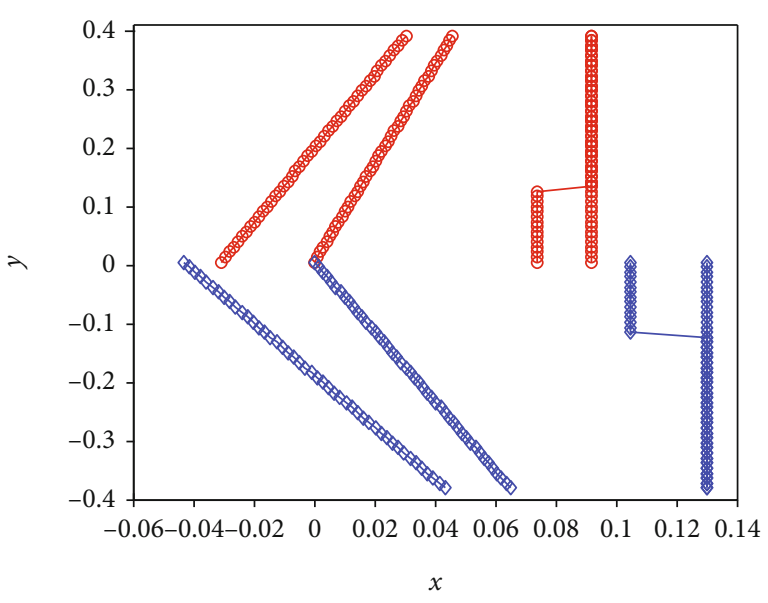

Figure 16: Wing orientation of the $\theta_{\text {Attach }}$ in the $X-Y$ coordinate.

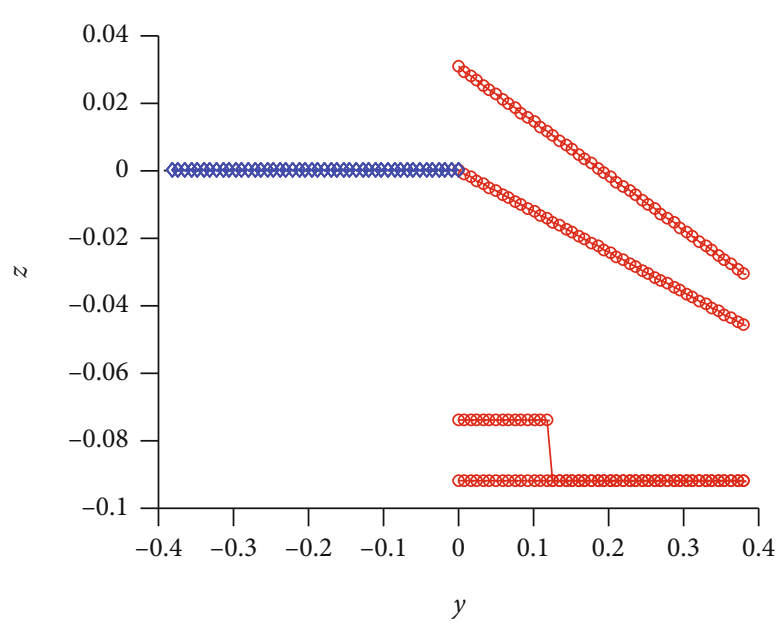

Figure 17: Wing orientation of the $\theta_{\text {Attach }}$ in the $Y-Z$ coordinate.

structed in C81 form using the table lookup method. A uniform inflow model or pitt-peter (dynamic inflow) models is selectively applied to the rotor inflow model as depending on the rotor type, analysis efficiency, and various applications [15].

The kinematics at a blade element has to be defined to express the rotor dynamics. The coordinate of the rotorcraft is defined as shown in Figure 7, and the basic velocity, acceleration, and angular velocity are expressed as Eqs. (3) and (4). The superscript $C I$ is used to define the vectors at the rotorcraft CG relative to the center of the inertial reference frame, whereas the subscript represents the frame ( $C$ for CG-fixed frame) used to define vector components. For instance, the vector $\boldsymbol{\omega}_{C}^{C I}$ is the angular velocity of the CGfixed frame relative to inertia frame, which is expressed on the CG-fixed frame. Figure 7 shows the front view of the rotor fixed nonrotating coordinates $\left(x_{H}, y_{H}, z_{H}\right)$ with rotational speed $\Omega$ in the CW direction.

$$
\begin{gathered}
\dot{\mathbf{r}}_{C}^{C I}=\mathbf{v}_{C}^{C I}=\left[\begin{array}{c}
u \\
v \\
w
\end{array}\right], \ddot{\mathbf{r}}_{C}^{C I}=\mathbf{a}_{C}^{C I}=\left[\begin{array}{c}
\dot{u}-r v+q w \\
\dot{v}-p w+r u \\
\dot{w}-q u+p v
\end{array}\right], \\
\boldsymbol{\omega}_{C}^{C I}=\left[\begin{array}{c}
p \\
q \\
r
\end{array}\right], \dot{\boldsymbol{\omega}}_{C}^{C I}=\left[\begin{array}{c}
\dot{p} \\
\dot{q} \\
\dot{r}
\end{array}\right], \boldsymbol{\omega}_{C}^{C I} \times=\tilde{\boldsymbol{\omega}}_{C}^{C I}=\left[\begin{array}{ccc}
0 & -r & q \\
r & 0 & -p \\
-q & p & 0
\end{array}\right] .
\end{gathered}
$$

The linear velocity and acceleration at the rotor center can be expressed by using the definition of the rotorcraft's CG position $\mathbf{r}_{C}^{C I}$ and the center of the rotor $\mathbf{r}_{C}^{H C}$ as shown in Equation (5). The subscript $R$ in Equation (5) means the rotating hub frame of rotor.

$$
\begin{aligned}
& \dot{\mathbf{r}}_{R}^{R I}=\mathbf{L}_{R C} \dot{\mathbf{r}}_{C}^{C I}+\mathbf{L}_{R C} \tilde{\boldsymbol{\omega}}_{C}^{C I} \mathbf{r}_{C}^{H C}, \\
& \ddot{\mathbf{r}}_{R}^{R I}=\mathbf{L}_{R C} \ddot{\mathbf{r}}_{C}^{C I}+\mathbf{L}_{R C}\left(\dot{\tilde{\boldsymbol{\omega}}}_{C}^{C I}+\tilde{\boldsymbol{\omega}}_{C}^{C I} \tilde{\boldsymbol{\omega}}_{C}^{C I}\right) \mathbf{r}_{C}^{H C} .
\end{aligned}
$$




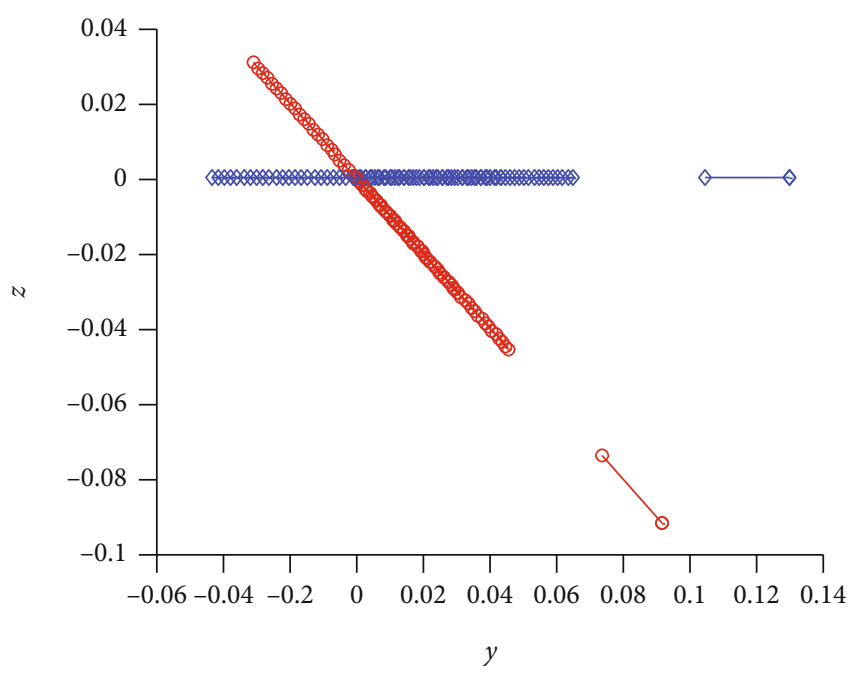

FIGURE 18: Wing orientation of the $\theta_{\text {Attach }}$ in the $X-Z$ coordinate.

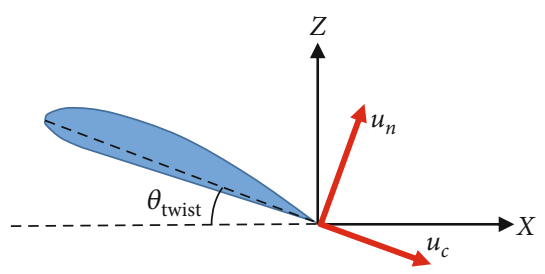

FIGURE 19: General airfoil configuration.

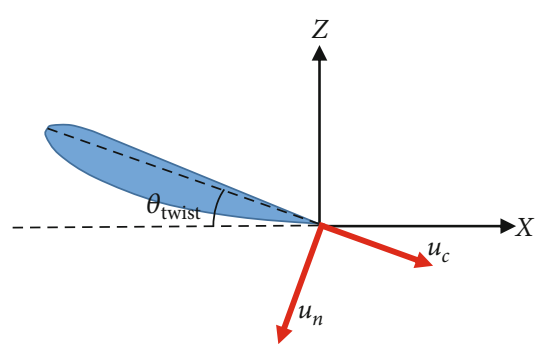

Figure 20: Upside-down airfoil configuration.

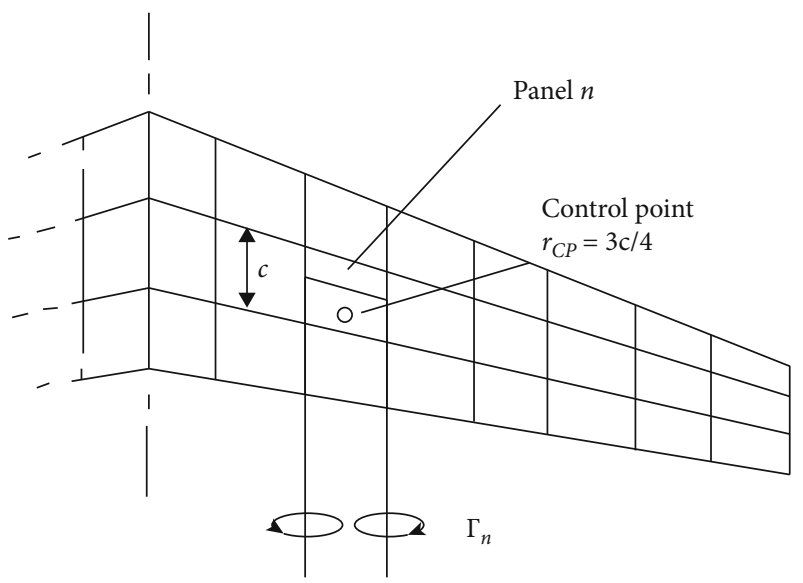

Figure 21: Concept of the vortex lattice method [16].

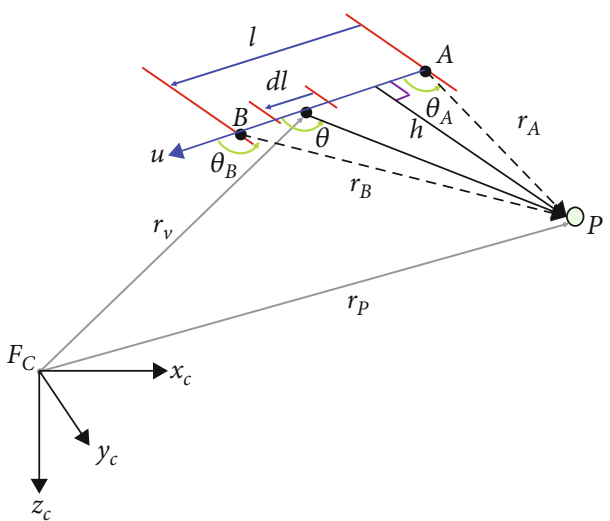

FIGURE 22: The relationship between the vortex filament and the control point based on biot-sarvart law [16].

The equations of the rotor dynamics have to reflect the order of the hinge changes, adding, and subtraction. It means that the rotor dynamics cannot be expressed by one formula without constraints. This study utilizes the generalized vector kinematics [7]. The generalized vector kinematics is derived by relative motion from several coordinate systems as shown in Figure 7. Also, the generalized vector kinematics is utilized to derive the implicit formulation of the rotor motion equations. It can be applied regardless of the shape and arrangement order of various types of rotor. The accuracy of the derived rotor motion equation can be easily verified. The linear velocity and acceleration are described at the lead-lag hinge because the feathering motion is not applied to rotor dynamics in this study. Moreover, the lead-lag hinge is the outer most hinge as shown in Figure 5. It is decided to denote the variables related in feathering motion in Equations (6) and (7) for convenience. The linear velocity and acceleration at the lead-lag hinge for blade section can be expressed as Equations (6) and (7), respectively, where the subscripts $L$ and $T$ mean the lead-lag hinge and the blade section frame, respectively. Furthermore, the linear velocity and 

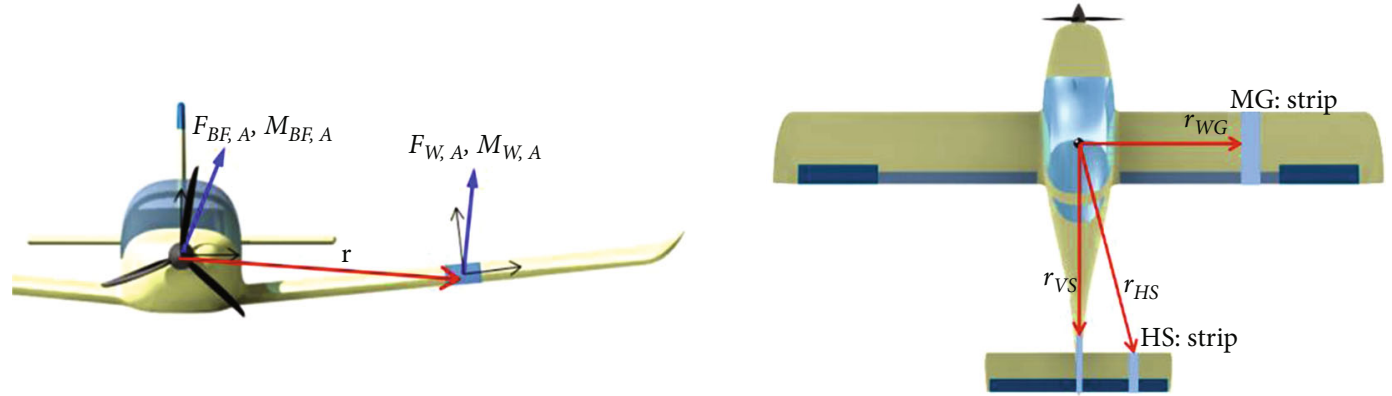

VS: strip

Figure 23: Concept of strip theory [16].

acceleration are defined on CG and AC of the blade section to reflect the rotor dynamics and to calculate the aerodynamic forces, respectively.

$$
\begin{aligned}
& \dot{\mathbf{r}}_{L}^{T I}=\mathbf{L}_{L R} \dot{\mathbf{r}}_{R}^{R I}+\mathbf{L}_{L R} \tilde{\boldsymbol{\omega}}_{R}^{R I} \mathbf{L}_{R L} \\
& \cdot\left\{\left(l_{P} \mathbf{L}_{L R}+l_{S} \mathbf{L}_{L P}+l_{F} \mathbf{L}_{L S}+l_{L} \mathbf{L}_{L F}+l_{A}\right) \mathbf{e}_{X}\right\} \\
& \cdot\left[-\dot{\beta} \mathbf{L}_{L S} \mathbf{E}_{2} \mathbf{L}_{S L}\left(l_{L} \mathbf{L}_{L F}+l_{A}\right) \mathbf{e}_{X}-\sigma \dot{\zeta} \mathbf{L}_{L F} \mathbf{E}_{3} \mathbf{L}_{F L} l_{A} \mathbf{e}_{X}\right. \\
& +\left\{\begin{array}{c}
\mathbf{L}_{L R} \tilde{\boldsymbol{\omega}}_{R}^{R I} \mathbf{L}_{R L}-\dot{\beta} \mathbf{L}_{L S} \mathbf{E}_{2} \mathbf{L}_{S L} \\
-\sigma \dot{\zeta} \mathbf{L}_{L F} \mathbf{E}_{3} \mathbf{L}_{F L}+\sigma \dot{\theta} \mathbf{E}_{1}
\end{array}\right\} \\
& \left.\cdot\left(r_{B} \mathbf{L}_{L A} \mathbf{e}_{X}-\sigma y_{S} \mathbf{L}_{L T} \mathbf{e}_{Y}-z_{A} \mathbf{L}_{L T} \mathbf{e}_{Z}\right)\right] \text {, } \\
& \ddot{\mathbf{r}}_{L}^{T I}=\mathbf{L}_{L R} \ddot{\mathbf{r}}_{R}^{R I}+\mathbf{L}_{L R}\left(\dot{\tilde{\boldsymbol{\omega}}}_{R}^{R I}+\tilde{\boldsymbol{\omega}}_{R}^{R I} \tilde{\boldsymbol{\omega}}_{R}^{R I}\right) \mathbf{L}_{R L}\left(l_{P} \mathbf{L}_{L R}+l_{S} \mathbf{L}_{L P}+l_{F} \mathbf{L}_{L S}\right) \mathbf{e}_{X} \\
& +\left\{\mathbf{L}_{L R}\left(\dot{\tilde{\boldsymbol{\omega}}}_{R}^{R I}+\tilde{\boldsymbol{\omega}}_{R}^{R I} \tilde{\boldsymbol{\omega}}_{R}^{R I}\right) \mathbf{L}_{R L}+\mathbf{L}_{L S}\left(-\ddot{\beta} \mathbf{I}+\dot{\beta}^{2} \mathbf{E}_{2}\right) \mathbf{E}_{2} \mathbf{L}_{S L}\right. \\
& +\mathbf{L}_{L F}\left(-\sigma \ddot{\zeta} \mathbf{I}+\dot{\zeta}^{2} \mathbf{E}_{3}\right) \mathbf{L}_{F L} \mathbf{E}_{3}+2 \sigma \dot{\beta} \dot{\zeta} \mathbf{L}_{L S} \mathbf{E}_{2} \mathbf{L}_{S L} \mathbf{E}_{3} \\
& \left.+\mathbf{L}_{L R} \tilde{\boldsymbol{\omega}}_{R}^{R I} \mathbf{L}_{R L}\left(-2 \dot{\beta} \mathbf{L}_{L S} \mathbf{E}_{2} \mathbf{L}_{S L}-2 \sigma \dot{\zeta} \mathbf{E}_{3}\right)\right\} r_{B} \mathbf{e}_{X} \text {. }
\end{aligned}
$$

The velocity $\dot{\mathbf{r}}_{L}^{T I}$ is composed by three parts such as linear velocity, angular velocity from lead-lag hinge, and effect of the angular rate from lead-lag hinge to the blade section. Although the acceleration as Equation (7) can be derived by using similar way to define Equation (6), the only difference between Equation (7) and Equation (6) is that Equation (6) considers the sweep length $y_{S}$ and an hedral length $z_{A}$. The variable $\sigma$ denotes the rotating direction which is CW rotation $(\sigma=-1)$ and CCW rotation $(\sigma=1)$.

The flap, lead-lag, and RPM dynamics associated with rotor motion are defined as Equations (8)-(10), respectively. Each dynamics can be selectively used by rotor type. If the rotor type is defined as the propeller, the rotor motion does not apply as noticed in Table 1, whereas if the general rotor is selected, the rotor motion is used. Moreover, the relative angles such as flapping angles are computed with each rotor motion. The flapping motion occurs of the $y$-axis associated with rotating rotor hub frame because of the flap hinge. The unit vector of $y$-axis and $z$-axis on rotor hub frame is defined as $\mathbf{e}_{Y}$ and $\mathbf{e}_{Z}$, respectively. The lead-lag and RPM

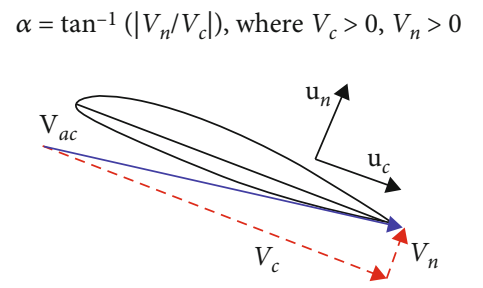

Figure 24: AOA with $V_{c}>0, V_{n}>0$.

$$
\alpha=-\tan ^{-1}\left(\left|V_{n} / V_{c}\right|\right) \text {, where } V_{c}>0, V_{n}<0
$$

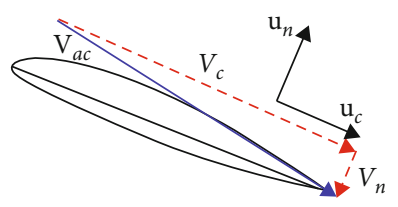

FIGURE 25: AOA with $V_{c}>0, V_{n}<0$. $\alpha=\pi / 2+\tan ^{-1}\left(\left|V_{n} / V_{c}\right|\right)$, where $V_{c}<0, V_{n}>0$

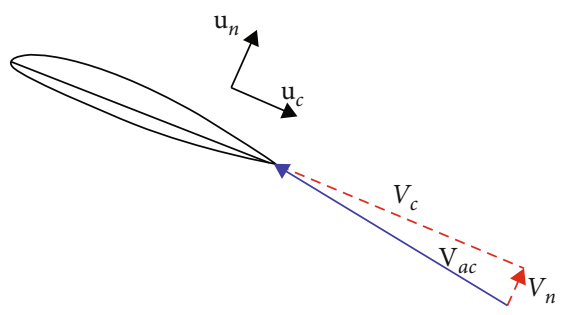

Figure 26: AOA with $V_{c}<0, V_{n}>0$.

$\alpha=-\pi / 2+\tan ^{-1}\left(\left|V_{n} / V_{c}\right|\right)$, where $V_{c}<0, V_{n}<0$

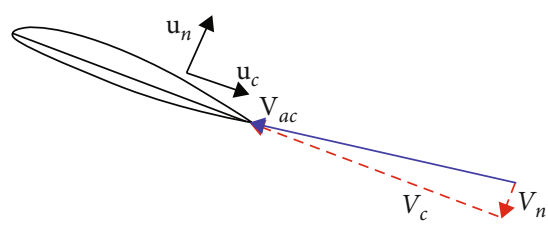

Figure 27: AOA with $V_{c}<0, V_{n}<0$.

dynamics move on the $z$-axis as the same principle of flapping motion. The flapping motion and RPM dynamics are needed to consider rotating direction additionally. The 
RPM dynamics does not consider the damping ratio and spring constant because the rotor shaft linked directly to the gear box and engine. So, the inertia forces in Equation (10) are divided as two forces generated by acceleration at the blade section and shaft $I_{D R V} \dot{\Omega}$, respectively. The variables which use in the following equations are listed in Table 3.

$$
\begin{gathered}
\int m_{B} \overline{\mathbf{d}}_{F} \times\left(\mathbf{L}_{F L} \ddot{\mathbf{r}}_{L}^{T I}-\mathbf{L}_{F C} \mathbf{g}_{C}^{C I}\right) \cdot \mathbf{e}_{Y} d r_{B}-C_{\dot{\beta}} \dot{\beta}-K_{\beta} \beta \\
=\int\left(\mathbf{L}_{F L} d \mathbf{M}_{\mathrm{Sec}}^{\text {Aero }}+\mathbf{d}_{F} \times \mathbf{L}_{F L} d \mathbf{F}_{\mathrm{Sec}}^{\text {Aero }}\right) \cdot \mathbf{e}_{Y} \\
\sigma \int m_{B} \overline{\mathbf{d}}_{L} \times\left(\ddot{\mathbf{r}}_{L}^{T I}-\mathbf{L}_{L C} \mathbf{g}_{C}^{C I}\right) \cdot \mathbf{e}_{Z} d r_{B}-C_{\dot{\zeta}} \dot{\zeta}-K_{\zeta} \zeta \\
=\sigma \int\left(d \mathbf{M}_{\mathrm{Sec}}^{\text {Aero }}+\mathbf{d}_{L} \times d \mathbf{F}_{\mathrm{Sec}}^{\text {Aero }}\right) \cdot \mathbf{e}_{Z} \\
\left.\sum_{J=1}^{N_{b}} \sigma \int m_{B} \overline{\mathbf{d}}_{R} \times\left(\mathbf{L}_{R L} \ddot{\mathbf{r}}_{L}^{T I}-\mathbf{L}_{R C} \mathbf{g}_{C}^{C I}\right) \cdot \mathbf{e}_{Z} d r_{B}\right\}+I_{D R V} \dot{\Omega} \\
\left.=\sum_{J=1}^{N_{b}} \sigma \iint\left(\mathbf{L}_{R L} d \mathbf{M}_{\mathrm{Sec}}^{\text {Aero }}+\mathbf{d}_{R} \times \mathbf{L}_{R L} d \mathbf{F}_{\text {Sec }}^{\text {Aero }}\right) \cdot \mathbf{e}_{Z}\right\}+Q_{\text {Engine }}
\end{gathered}
$$

The aerodynamic forces $d \mathbf{F}_{\text {Sec }}^{\text {Aero }}$ and moments $d \mathbf{M}_{\text {Sec }}^{\text {Aero }}$ for the unit section on the blade have to be calculated to compute the flap, lead-lag, and RPM dynamics. Figure 8 shows the general information for generating aerodynamic forces with induced drag at the blade section. The aerodynamic forces generated by blade section can be defined as Equation (11).

$$
d \mathbf{F}_{L}^{\text {Aero }}=\left(\begin{array}{c}
d R \\
\sigma(-d L \sin \phi-d D \cos \phi) \\
d L \cos \phi-d D \sin \phi
\end{array}\right), d \mathbf{M}_{L}^{\text {Aero }}=\left(\begin{array}{c}
\sigma d M \\
0 \\
0
\end{array}\right)
$$

$$
\phi=\tan ^{-1}\left(\frac{U_{P}}{U_{T}}\right), \alpha=\theta-\phi=\theta-\tan ^{-1}\left(\frac{U_{P}}{U_{T}}\right),
$$

where $d R, d L, d D$, and $d M$ are the spanwise axial forces, lift, drag, and moment on the unit section for blade,respectively, to calculate the aerodynamic forces. These four variables are expressed as Equation (13).

$$
\begin{aligned}
& d L=\frac{1}{2} C_{L} \rho\left(U_{T}^{2}+U_{P}^{2}\right) c d r_{B}, d D=\frac{1}{2} C_{D} \rho\left(U_{T}^{2}+U_{P}^{2}\right) c d r_{B} \\
& d R=\frac{1}{2} C_{R} \rho\left(U_{T}^{2}+U_{P}^{2}\right) c d r_{B}, d M=\frac{1}{2} C_{D} \rho\left(U_{T}^{2}+U_{P}^{2}\right) c^{2} d r_{B} .
\end{aligned}
$$

Here, $c$ is the distance of the camber for blade. $C_{L}, C_{D}$, and $C_{R}$ are the aerodynamic coefficients for lift, drag, and spanwise axial force, respectively. The coefficients are estimated using C81 tables by table-lookup technique. Especially, the table-lookup technique can change the

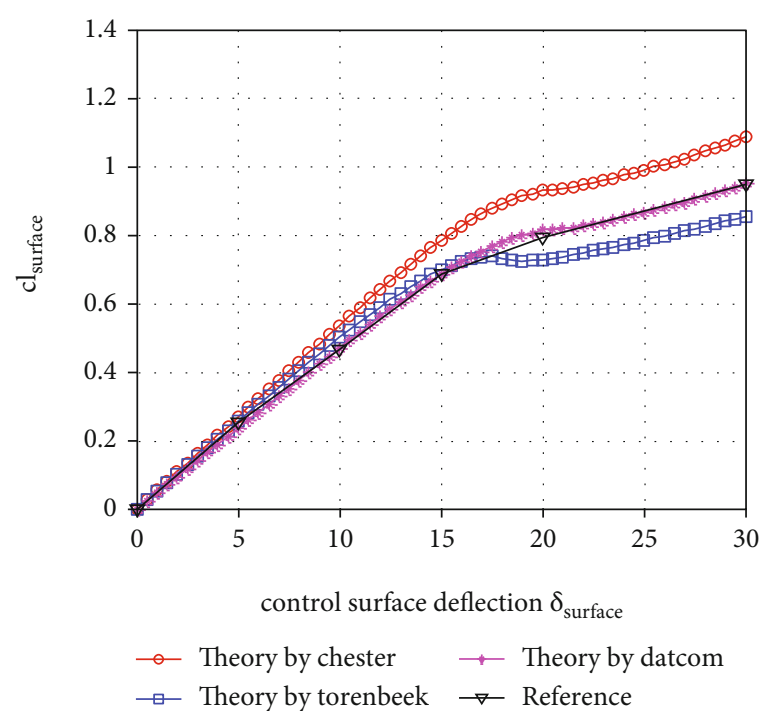

FIGURE 28: Comparison results for lift coefficient computed by several theories and wind tunnel data [21].

TABLE 4: The specification associated with wing model.

\begin{tabular}{lc}
\hline Variable & Description \\
\hline Airfoil & NACA23012 \\
$t / c$ & 0.12 \\
$c_{\text {surface }}$ & $0.2 c_{\text {airfoil }}$ \\
$C_{l_{\alpha}}$ & $0.1080(/ \mathrm{deg})$ \\
AOA & $6.1879(/ \mathrm{rad})$ \\
Reynolds number & $0(\mathrm{deg})$ \\
Deflection of control surface & $8.0 \times 10^{6}$ \\
\hline
\end{tabular}

coefficients when the blade has the conversion section. The velocity component to generate the aerodynamic forces and moment can be defined as follow.

$$
\left[\begin{array}{c}
U_{R} \\
U_{T} \\
U_{P}
\end{array}\right]=\left[\begin{array}{c}
V_{L X} \\
V_{L Y} \\
V_{L Z}
\end{array}\right]=\mathbf{v}_{L}^{T I}-\mathbf{v}_{L}^{\text {tur }}-\mathbf{v}_{L}^{\text {ind }}=\mathbf{v}_{L}^{\text {aero }}
$$

$\mathbf{v}_{L}^{\mathrm{TI}}, \mathbf{v}_{L}^{\text {tur }}$, and $\mathbf{v}_{L}^{\text {ind }}$ mentioned in Equation (14) are $\dot{\mathbf{r}}_{L}^{\mathrm{TI}}$, turbulence, and induced inflow, respectively.

2.2. Unified Wing Modeling: Geometry. The wings have various shapes and are usually attached to the center of the fuselage for aircraft. The wings are classified by mounted position and purpose of control surface to construct a unified wing model that can reflect all characteristics of the wings.

A typical fixed wing aircraft is equipped with five wings such as the left and right main wings, the left and right horizontal stabilizers, and the vertical stabilizer. However, a compound rotorcraft can be made with wings at various angles and positions. This is the reason why the unified wing modeling technique has to be developed. The unified wing 


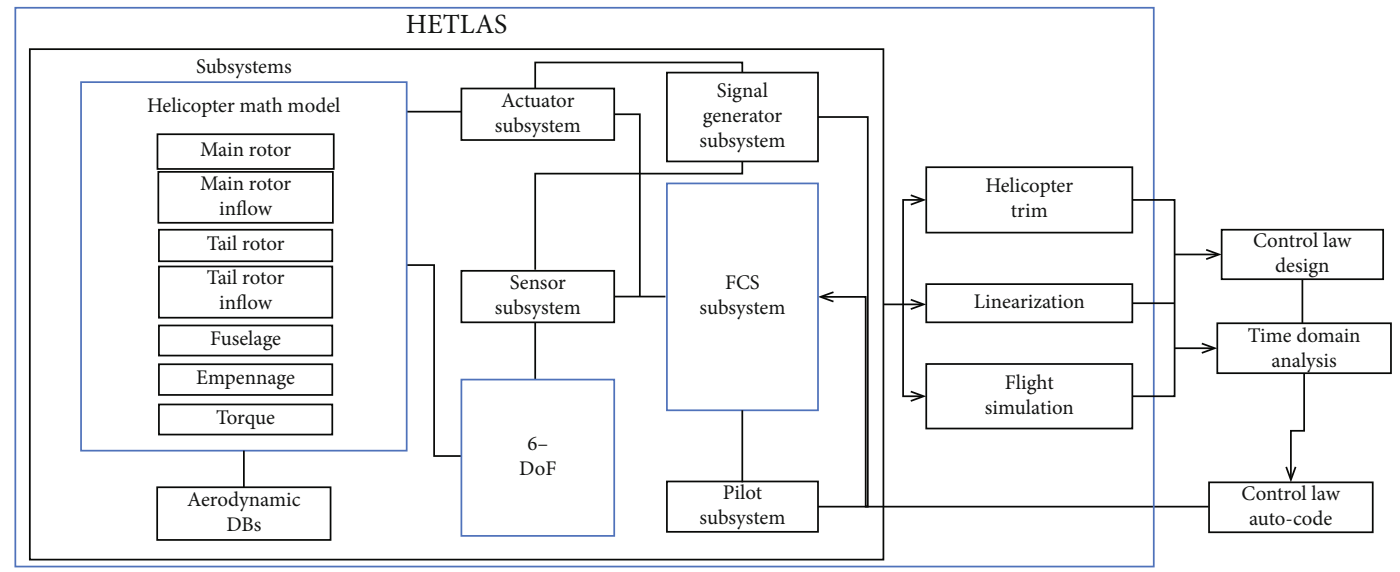

FIGURE 29: The structure of the HETLAS program [18].

model is capable of reflecting various wing shapes and positions. The mounting angle is based on the starboard side wing, and the wing coordinate is the same as the body coordinate of the aircraft. Three different angles are defined to represent the wing mounting angles such as elevation angle $\theta_{\text {Elev }}$, attachment angle $\theta_{\text {Attach }}$, and rotation angle $\theta_{\text {Rot }} . \theta_{\text {Elev }}$ rotates in the CW direction of the $x$-axis, and $\theta_{\text {Attach }}$ rotates in the CCW direction of the $y$-axis. Finally, $\theta_{\text {Rot }}$ rotates in the CW direction of the $z$-axis. The $x$-, $y$-, and $z$-axis coordinate rotation matrices (active transformation matrix) defined in Equation (1) are applied for the rotation of the wing. Thus, the wing coordinate rotation matrix $\mathbf{L}_{\mathrm{WING}}$ using Equation (1) is defined as given in Equation (15).

$$
\mathbf{L}_{\mathrm{WING}}=\mathbf{L}_{1}\left(\theta_{\text {Elev }}\right) \mathbf{L}_{2}\left(\theta_{\text {Attach }}\right) \mathbf{L}_{3}\left(\theta_{\text {Rot }}\right)
$$

The unified wing model can handle the angle of elevation, attachment, and rotation angle for wing. The elevation angle $\theta_{\text {Elev }}$ and the attachment angle $\theta_{\text {Attach }}$ for wing can be defined as shown in Figures 9 and 10. The fuselage line and the starboard side of the wing are the standard line to define each angle. Figures 11-14 show the results of wing orientation for elevation angle $\theta_{\text {Elev }}$. The blue color expresses the port wing, and the red color means the starboard wing in Figures 11-14. Figure 11 shows the wing orientations on the $X-Y-Z$ coordinate when the elevation angle for starboard side wing and port side wing are defined as 45 (deg) and 0 (deg), respectively. Moreover, the attachment angles are same as 0 (deg) for port side and starboard side. Figures $12-14$ are expressing the wing orientation results on the $X-Y, Y-Z$, and $X-Z$ coordinate, respectively. This is the very useful function to make the geometry for various compound aircraft.

Figures 15-18 show the wing geometry when the attachment angles of starboard side and port side wing are defined as 45 (deg) and 0 (deg). The difference between the starboard side and the port side is expressed on the $X-Y-Z$ coordinate in Figure 15. The attachment angles for each wing can be
TABLE 5: The configuration data for reference helicopter [19].

\begin{tabular}{lc}
\hline Configurations & Data \\
\hline Length & $19.0(\mathrm{~m})$ \\
Rotor diameter & $15.8(\mathrm{~m})$ \\
Height & $4.5(\mathrm{~m})$ \\
Maximum takeoff weight & $8,709(\mathrm{~kg})$ \\
Engine & $1,855 \times 2(\mathrm{SHP})$ \\
Maximum cruise speed & $279(\mathrm{~km} / \mathrm{h})$ \\
\hline
\end{tabular}

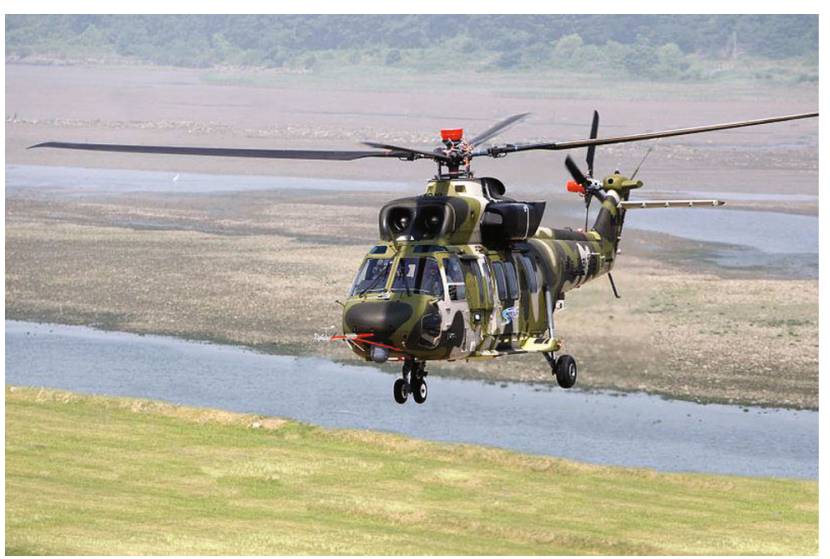

FIgURE 30: The shape of the reference helicopter [19].

TABLE 6: The configuration data for the BO-105 helicopter [20].

\begin{tabular}{lc}
\hline Configurations & Data \\
\hline Length & $11.8(\mathrm{~m})$ \\
Rotor diameter & $9.8(\mathrm{~m})$ \\
Height & $3.0(\mathrm{~m})$ \\
Maximum takeoff weight & $2,500(\mathrm{~kg})$ \\
Engine & $420 \times 2(\mathrm{SHP})$ \\
Maximum cruise speed & $268(\mathrm{~km} / \mathrm{h})$ \\
\hline
\end{tabular}




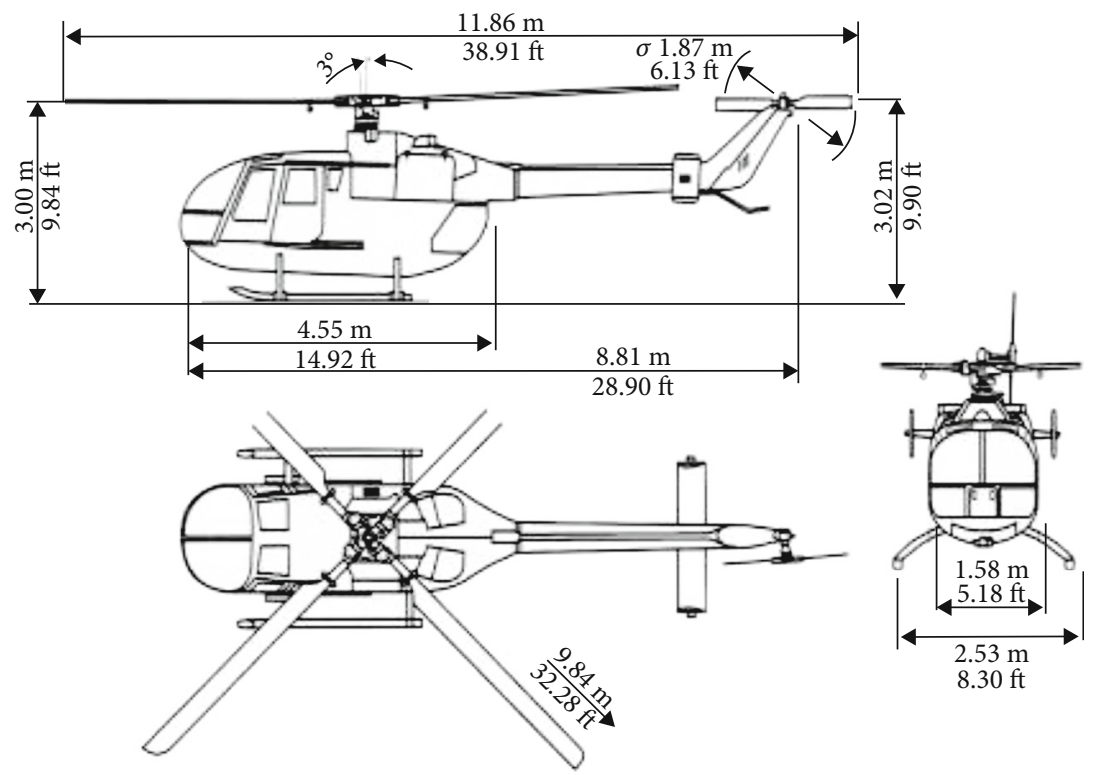

Figure 31: The shape of the BO-105 helicopter [20].

independently defined. Thus, the unified wing model can express all type geometries for compound aircraft.

Finally, the unified wing model considers the upsidedown configuration of airfoil. This configuration is mostly applied to horizontal stabilizer. The horizontal stabilizer is attached to generate a positive pitching moment. It is the same as when the direction of the normal vector of the airfoil is reversed. Figures 19 and 20 show the normal vector $\mathbf{u}_{n}$ and tangential vector $\mathbf{u}_{c}$ on the $X-Z$ airfoil coordinate.

$\mathbf{u}_{n}$ and $\mathbf{u}_{c}$ on the wing coordinate as shown in Figures 9 and 10 can be defined as Equation (16), where $\mathbf{e}_{x}, \mathbf{e}_{y}, \mathbf{e}_{z}$ are unit vectors of each axis on the wing model.

$$
\begin{aligned}
\mathbf{u}_{n}=\mathbf{L}_{\text {twist }} \mathbf{e}_{z} & =\left(\begin{array}{ccc}
\cos \theta_{\text {twist }} & 0 & -\sin \theta_{\text {twist }} \\
0 & 1 & 0 \\
\sin \theta_{\text {twist }} & 0 & \cos \theta_{\text {twist }}
\end{array}\right)\left(\begin{array}{l}
0 \\
0 \\
1
\end{array}\right) \\
& =\left(\begin{array}{c}
\sin \theta_{\text {twist }} \\
0 \\
\cos \theta_{\text {twist }}
\end{array}\right), \mathbf{u}_{c}=\mathbf{L}_{\text {twist }} \mathbf{e}_{x} \\
& =\left(\begin{array}{ccc}
\cos \theta_{\text {twist }} & 0 & -\sin \theta_{\text {twist }} \\
0 & 1 & 0 \\
\sin \theta_{\text {twist }} & 0 & \cos \theta_{\text {twist }}
\end{array}\right)\left(\begin{array}{l}
1 \\
0 \\
0
\end{array}\right) \\
& =\left(\begin{array}{c}
\cos \theta_{\text {twist }} \\
0 \\
-\sin \theta_{\text {twist }}
\end{array}\right) .
\end{aligned}
$$

The upside-down airfoil configuration changes a direction of normal vector $\mathbf{u}_{n}$ as shown in Equation (17). The aerodynamic forces and moments can be easily computed by Equation (17).

$$
\begin{aligned}
\mathbf{u}_{n, \text { upside_down }} & =\mathbf{L}_{2}(\pi) \mathbf{u}_{n} \\
& =\left(\begin{array}{ccc}
-1 & 0 & 0 \\
0 & 1 & 0 \\
0 & 0 & -1
\end{array}\right)\left(\begin{array}{c}
\sin \theta_{\text {twist }} \\
0 \\
\cos \theta_{\text {twist }}
\end{array}\right) \\
& =\left(\begin{array}{c}
-\sin \theta_{\text {twist }} \\
0 \\
-\cos \theta_{\text {twist }}
\end{array}\right) .
\end{aligned}
$$

2.3. Unified Wing Modeling: Aerodynamic Forces and Moments. The important variable is a wind velocity to compute the lift and drag for main wing, horizontal, and vertical stabilizer. The wind velocity is defined by true air speed and induces velocity. This study calculates the induced velocity to enhance the fidelity of the wing model. VLM is used to compute the induced velocity. Figure 21 expresses the concept of the vertex lattice method.

The horse shoe vortex and the control point $P$ are applied to each lattice. A number of lattice can be defined by designer. The control point $P$ is located on the $50 \%$ point of spanwise direction and the $75 \%$ point of chord direction, respectively. The induced velocities of each lattice on the main wing and fin stabilizer are calculated by Biot-Savart law as shown in Figure 22. The Biot-Savart law is expressed to Equation (18). 

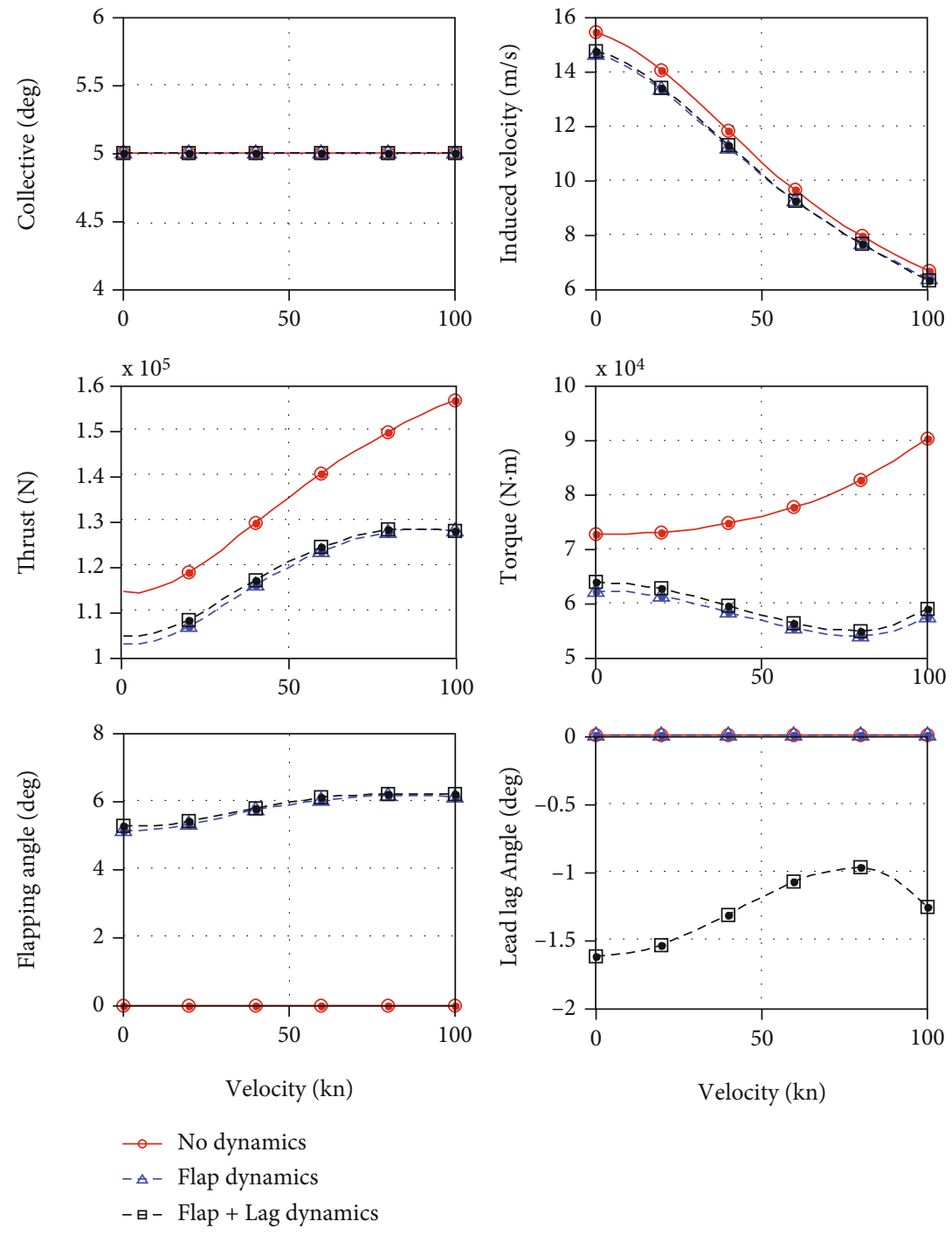

FIGURE 32: Results of rotor performance analysis.

$$
\begin{aligned}
d \mathbf{V}_{\text {ind }}\left(\mathbf{r}_{P}\right) & =\frac{\Gamma}{4 \pi} \frac{d \mathbf{l} \times \mathbf{r}}{\|\mathbf{r}\|^{3}}=\frac{\Gamma}{4 \pi} \frac{d \mathbf{l} \times\left(\mathbf{r}_{P}-\mathbf{r}_{v}\right)}{\left\|\mathbf{r}_{P}-\mathbf{r}_{v}\right\|^{3}} \\
& =\Gamma_{c} \frac{h^{2}}{4 \pi \sqrt{r_{c}^{2}+h^{2}}} \frac{d \mathbf{l} \times\left(\mathbf{r}_{P}-\mathbf{r}_{v}\right)}{\left\|\mathbf{r}_{P}-\mathbf{r}_{v}\right\|^{3}} .
\end{aligned}
$$

$F_{C}$ means an origin point of the body coordinate. $\mathbf{r}_{P}$ and $\mathbf{r}_{v}$ are a distance vector from the origin point of the body coordinate to control point of each lattice and to unit vortex filament $d \mathbf{l}$, respectively. $\mathbf{r}_{c}$ is a core radius for vortex filament, and $h$ is a perpendicular distance from vertex filament to control point $P$. If the number of lattice is increased, the number of control point is expended. The lift of each lattice can be computed by Equation (19).

$$
L=\rho V \Gamma_{c}
$$

where $\rho$ and $V$ are the density of air and the true air speed, respectively. $\Gamma_{c}$ is the vortex strength. Kutta-
Joukowski theory as shown in Equation (20) is used to compute $\Gamma_{c}$.

$$
\Gamma_{c}=\frac{1}{2} C_{L} V s
$$

$C_{L}$ and $s$ are the lift coefficient and the area of unit section. Finally, the velocity $V$ has to be computed, and it is a $\left\|\mathbf{V}_{\text {ac }}\right\| . \mathbf{V}_{\text {ac }}$ is a velocity vector that the $Y$-component is zero. The velocity vector is calculated after computing $\mathbf{v}_{k} \cdot \mathbf{v}_{k}$ is a velocity vector including the $Y$-component on each lattice. The subscript of $\mathbf{v}_{k}$ is a number of lattice in the main wing and fin stabilizer. $\mathbf{v}_{k}$ is defined as Equation (21).

$$
\mathbf{v}_{k}=\mathbf{v}_{C}^{C I}+\tilde{\boldsymbol{\omega}}_{C}^{C I} \mathbf{r}_{C}^{P C}+\mathbf{v}_{\text {ind }, k}
$$

where $\mathbf{v}_{C}^{C I}$ is a true air speed vector associated with CG, and $\boldsymbol{\omega}_{C}^{C I}$ is a angular velocity vector of aircraft. $\mathbf{r}_{C}^{P C}$ is a distance vector between the CG of aircraft and the control point 

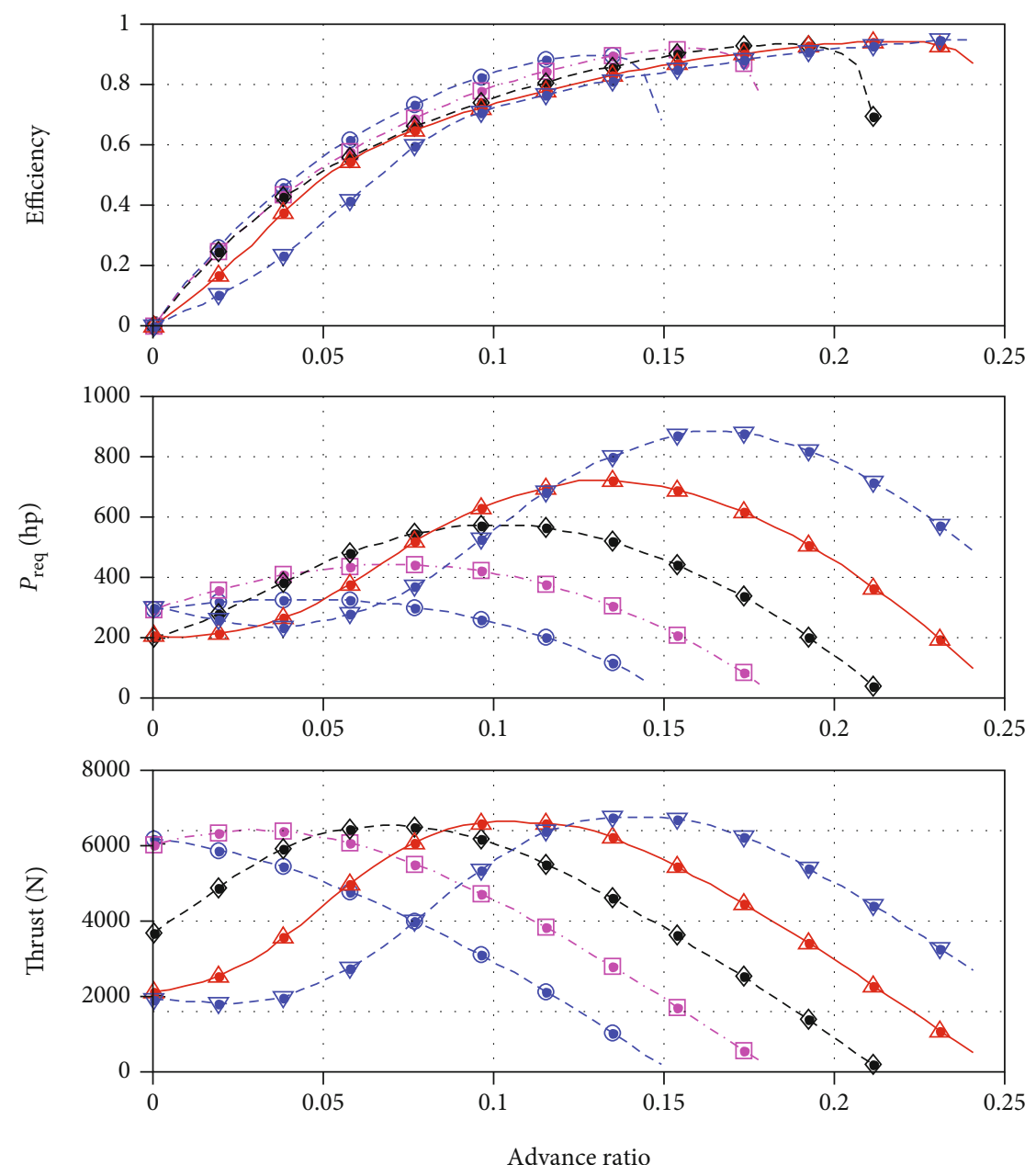

$$
\begin{aligned}
-\diamond-: \text { Col }-8 \mathrm{deg} & -\triangleleft: \mathrm{Col} 4 \mathrm{deg} \\
-\square-: \text { Col }-4 \mathrm{deg} & -\nabla-: \mathrm{Col} 8 \mathrm{deg} \\
-\diamond-: \text { Col } 0 \mathrm{deg} &
\end{aligned}
$$

FiguRE 33: Results of propeller performance analysis.

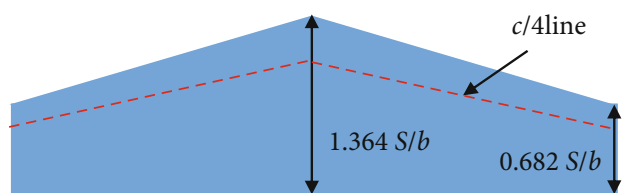

FIGURE 34: Configuration of tapered wing without control surface.

$P$ of each lattice. $\mathbf{v}_{\text {ind, } k}$ is a induced velocity vector of each lattice.

$$
\mathbf{V}_{\mathrm{ac}}=\mathbf{v}_{k}-\left(\mathbf{v}_{k} \cdot \mathbf{e}_{y}\right) \mathbf{e}_{y}=\mathbf{V}_{c}+\mathbf{V}_{n}=V_{c} \mathbf{u}_{c}+V_{n} \mathbf{u}_{n}
$$

$\mathbf{e}_{y}$ is defined as shown in Equation (23).

$$
\mathbf{e}_{y}=-\left(\mathbf{u}_{c} \times \mathbf{u}_{n}\right) .
$$

The calculated aerodynamic forces and moments for each lattice have to be expressed on the body coordinate. This study use the strip theory to incorporate the aerodynamic forces and moments on the origin point of the body coordinate. The CG is the same with the origin point of the body coordinate. Figure 23 shows the concept of strip theory.

Thus, the computed aerodynamic forces and moments of each lattice can be expressed on CG as shown in Equation (24). Here, $\alpha$ and $\beta$ are the AOA and the sideslip angle (SSA), respectively.

$$
\begin{aligned}
\mathbf{F}_{C}^{P C}=\mathbf{L}_{C P} \mathbf{F}_{P}^{P C} & =\left[\begin{array}{c}
-D \cos \alpha \cos \beta-Y \cos \alpha \sin \beta+L \sin \alpha \\
-D \sin \beta+Y \cos \beta \\
-D \sin \alpha \cos \beta-Y \sin \alpha \sin \beta-L \cos \alpha
\end{array}\right], \\
\mathbf{M}_{C}^{P C}=\mathbf{L}_{C P} \mathbf{M}_{P}^{P C}= & {\left[\begin{array}{c}
M_{x} \cos \alpha \cos \beta-M_{y} \cos \alpha \sin \beta-M_{z} \sin \alpha \\
M_{x} \sin \beta+M_{y} \cos \beta \\
M_{x} \sin \alpha \cos \beta-M_{y} \sin \alpha \sin \beta+M_{z} \cos \alpha
\end{array}\right] . }
\end{aligned}
$$



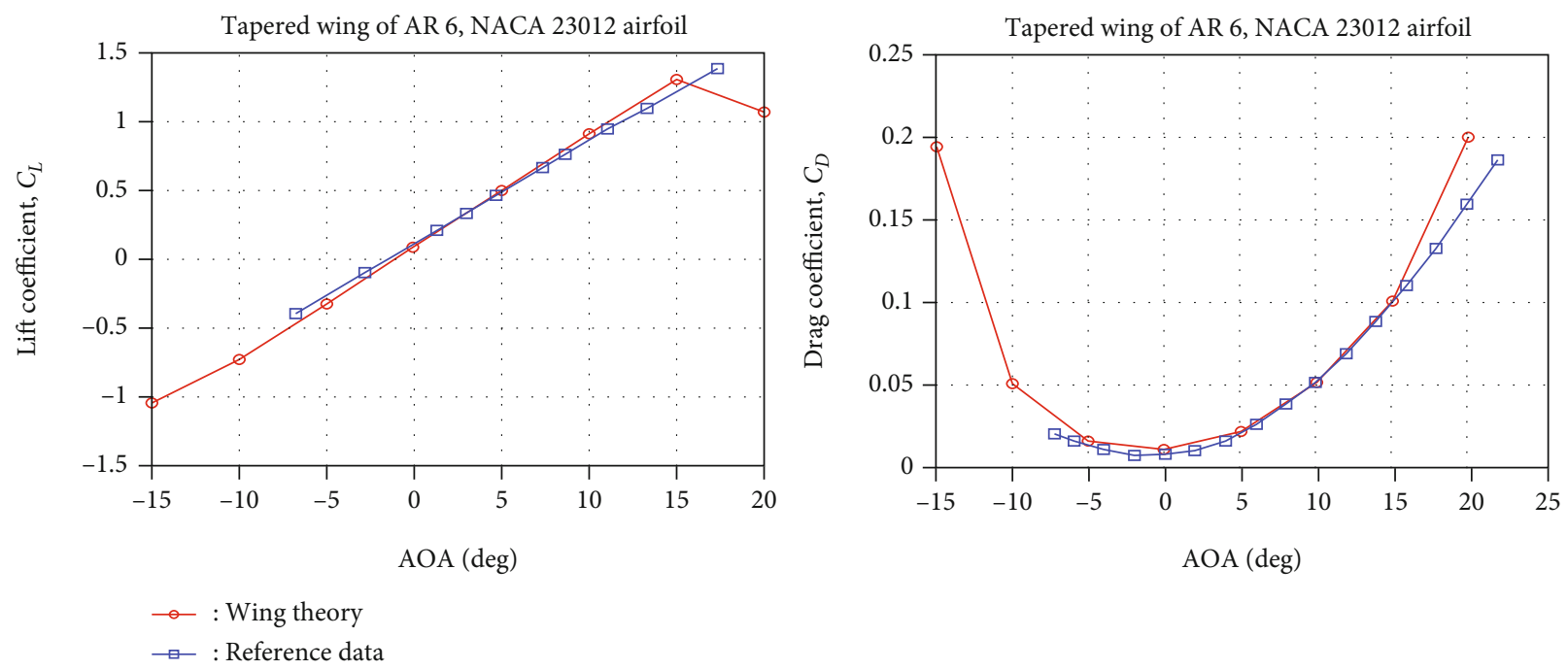

Figure 35: Comparison of the lift and drag coefficient.

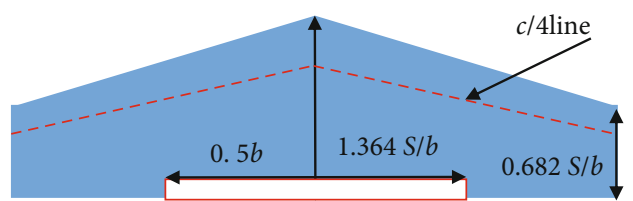

FIGURE 36: Configuration of tapered wing with control surface.

The aerodynamic forces and moments of main wing and fin stabilizers can be calculated by applying Equation (24) to all of lattice as shown in Equation (25).

$$
\begin{gathered}
\sum \mathbf{F}_{C}^{P C}=\sum_{j=1}^{n} \mathbf{L}_{C P} \mathbf{F}_{P_{j}}^{P C}, \\
\sum \mathbf{M}_{C}^{P C}=\sum_{j=1}^{n}\left(\mathbf{L}_{C P} \mathbf{M}_{P_{j}}^{P C}+\mathbf{r}_{C_{j}}^{P C} \times \mathbf{L}_{C P} \mathbf{F}_{P_{j}}^{P C}\right),
\end{gathered}
$$

where the subscript $j$ of the $P$ is order of lattice for main wing and fin stabilizer. So, $j$ th control point $P$ is described as $P_{j}$.

2.4. Unified Wing Modeling: Aerodynamic Coefficients. The AOA is calculated by 4 cases by using the absolute values of velocities. Each case is described as shown in Figures 24-27.

The influence of the wing lift coefficient on the control surface deflection is expressed as given in Equation (26). The control surface is considered as a plain flap. Here, $C_{l, \text { surface }}$ and $\delta_{\text {surface }}$ are the lift coefficient associated with control surface and control surface deflection angle, respectively.

$$
C_{l}=C_{l, 0}+C_{l_{\alpha}} \alpha+C_{l, \text { surface }} \delta_{\text {surface }} \cdot
$$

The main coefficient in Equation (26) is a $C_{l, \text { surface }}$ because other coefficients such as $C_{l, 0}$ and $C_{l_{\alpha}}$ are the constant or the functions associated with AOA, SSA, and Mach number. Thus, the calculation accuracy of $C_{l \text {,surface }}$ is the most important for the wing model. Several tools such as chester, torenbeek, and DATCOM have suggested the theory to calculate the coefficient $C_{l \text { surface }}$. A comparison of various empirical formulas calculating $C_{l \text {,surface }}$ indicates that the theory proposed in DATCOM is the most accurate as shown in Figure 28 [17]. The reference data in Figure 28 is the wind tunnel test data for NACA23012 airfoil. The specification of NACA23012 airfoil is described in Table 4. This study selects the theory proposed in DATCOM to compute $C_{l \text {,surface }}$.

\section{Application}

This section tries to validate the FDM built by the unified rotor and wing model. The single rotor helicopter and compound helicopter using coaxial rotor are used for checking fidelity of FDM. The helicopter models are used to the BO105 helicopter and the reference rotorcraft which is built by HETLAS. A reference rotorcraft is a Surion helicopter. HETLAS is a program for implementing trim, linearization, and simulation of the helicopter. Especially, this program had been validated by the Surion helicopter. Moreover, it has been already used to develop the FBW (fly-by-wire) system for Surion helicopter. Figure 29 shows the structure of the HETLAS program. The detail information of HETLAS is described in Ref. [15, 18].

The configuration data for reference helicopter are described as Table 5, and Figure 30 shows the shape of reference helicopter.

The configuration data and shape for the BO-105 helicopter are described as Table 6 and Figure 31, respectively.

The validation of proposed FDM would be conducted by comparing with analysis results computed from the HETLAS program. Trim analysis of the reference helicopter and simulation for the BO-105 rotorcraft in the time domain are used as the validation method for proposed FDM.

The trim analysis of the reference helicopter would be implemented by the HETLAS program and proposed FDM, where the validation of fidelity for proposed FDM is conducted by checking the difference of trim results between the HETLAS program and proposed FDM since the almost 

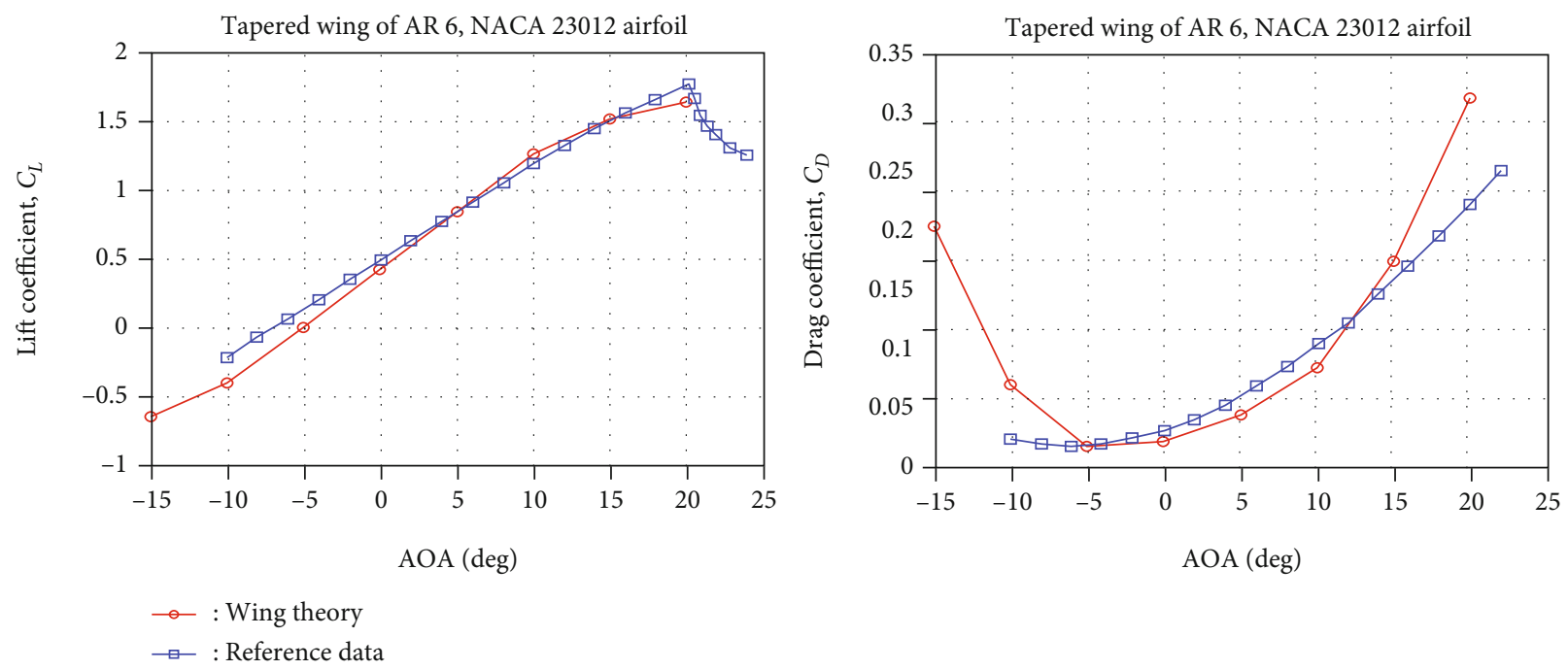

Figure 37: Comparison of the lift and drag coefficient with $\delta_{\text {surface }}=20.0(\mathrm{deg})$.

analysis and experimental data of reference helicopter are the confidential data. The simulation of the BO-105 helicopter would be performed by proposed FDM because the flight test data of the BO-105 helicopter has been opened. Especially, the AC-120 (advisory circular 120) criteria are applied to the simulation process to compare thoroughly.

3.1. Unified Rotor Model. This study shows that the rotor model employing the unified technique can be used to analyze various types of rotor dynamics. This study uses the rotor model validated by the HETLAS program. Figures 32 and 33 show the aerodynamic analysis results of the rotor and propeller using the unified rotor model.

The rotor of a typical helicopter is used for analyzing rotor performance as shown in Figure 32. The main rotor is used for analysis, and the collective inputs are defined as 5 degrees under the normal working states with increased forward speed. All results are compared with no rotor dynamics, flap dynamics, and flap and lag dynamics. The induced velocity has been decreased when the flight speed is raised. The induced velocity is inversely proportional to forward speed as $v_{i}=T / 2 \rho A V_{\infty}$ as understanding the momentum theory. The induced velocity is significantly decreased as forward speed increased as shown in Figures 32 and 33. Although the induced velocity is similar responses, the torques represent different behaviors. Torque with no dynamics is steadily increased, but the others have maintained those values. This is because the AOA of the blade section affects the drag force of the blade section. The drag coefficient at the blade section is reduced with a small effective AOA due to the increasing forward speed $U_{T}$ and the decreasing induced velocity $U_{P}$ as shown in Figure 8. This is the main reason to maintain the torque level. Furthermore, the flapping angles which can be considered as damping effect make the significant bias among all speed regions. The lag dynamics with negative lead-lag angles show less effect to torques increasing as a slightly bigger effective AOA with changing $U_{T}$.
The tractor-type propeller is used for analyzing propeller performance as shown in Figure 33 [7]. It shows the result of the analysis associated with advanced ratio and collective change. The thrusts and the required powers of the propeller have been increased when the advance ratio and collective input are raised, because the increments of flow velocity for propeller make the effective AOA of the blade section falls in the stall region. It means that the maximum thrust level is almost constant when the effective AOA is in the stall region. Furthermore, the propeller efficiency can be reached to maximum point.

Figures 32 and 33 have showed that the rotor dynamics can be selectively applied. Therefore, it is judged that the unified rotor model is highly effective to calculate the aerodynamic forces of various types of rotor or propeller.

3.2. Unified Wing Model. This research tries to validate the fidelity of the wing model. The tapered wing is used for checking the fidelity of the wing model. The specification of tapered wing without control surface is defined as Figure 34. The airfoil is a NACA 23012, and the wind velocity $\|\mathbf{V}\|$ is a $21.34(\mathrm{~m} / \mathrm{s})$.

Figures 35 is a lift coefficient and drag coefficient. These two aerodynamic coefficients computed by the wing model are very similar with reference data. The reference data is a wind tunnel test data for tapered wing.

The coefficients computed by the unified wing model for lift and drag are very similar with reference data. Thus, it is judged that the fidelity of the proposed wing model is appropriate. The specification of tapered wing with control surface is defined as Figure 36. The deflection angle of control surface is defined as 20.0 (deg). Other conditions are same with prior case.

Figures 37 show the comparison results of the lift and drag coefficients. The lift coefficient computed by the unified wing model is highly accurate with reference data that is measured by wind tunnel. Also, the drag coefficient 

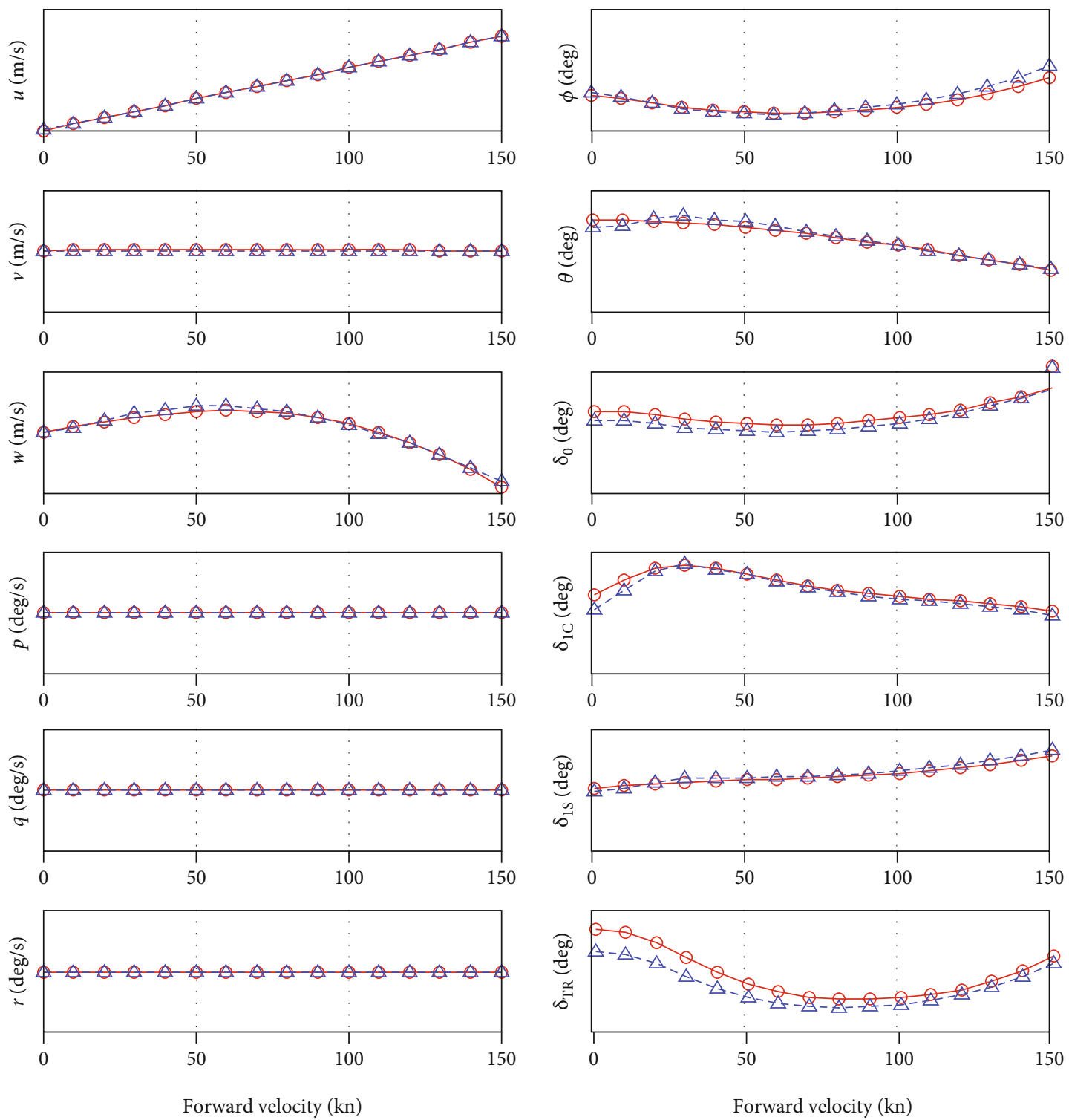

$\rightarrow$ Developed flight dynamics model

$-\Delta-$ Hetlas program

FIGURE 38: Comparisons of a typical model trim results (HETLAS vs proposed FDM).

calculated by the unified wing model is accurately computed. It is judged that the proposed wing modeling technique is highly accurate.

3.3. Single Rotor Helicopter. The helicopter using single rotor is choose to check the fidelity of FDM. FDM has been built as level 2. The reference helicopter validated by the HETLAS program is used to compare the accuracy of the proposed FDM technique. Figure 38 shows the trim analysis result calculated by HETLAS and proposed FDM. The results of HETLAS and FDM are indicated as blue and red, respectively. The detailed data for the reference helicopter used by HETLAS are confidential information. Thus, this paper mentions the difference of trim results computed by HETLAS and proposed FDM. All state variables such as flight velocity, angular rate, and attitude angle for FDM are very similar with HETLAS. However, the control variables are different. Those distinctions are about 0.5 degrees because the unified rotor model in FDM has enhanced the coefficient estimation on the sweep region of airfoil for blade section. Therefore, it is judged that the proposed technique to build FDM is very useful and accurate.

Also, Figures 39-43 describe the FDM validation results with flight test data in forward flight [22]. The validation is performed by the BO-105 helicopter and its flight test data. This validation is checked to meet helicopter simulator qualification [23]. Table 7 shows the tolerances with control inputs, attitude angles, and handling qualities in each axis. 

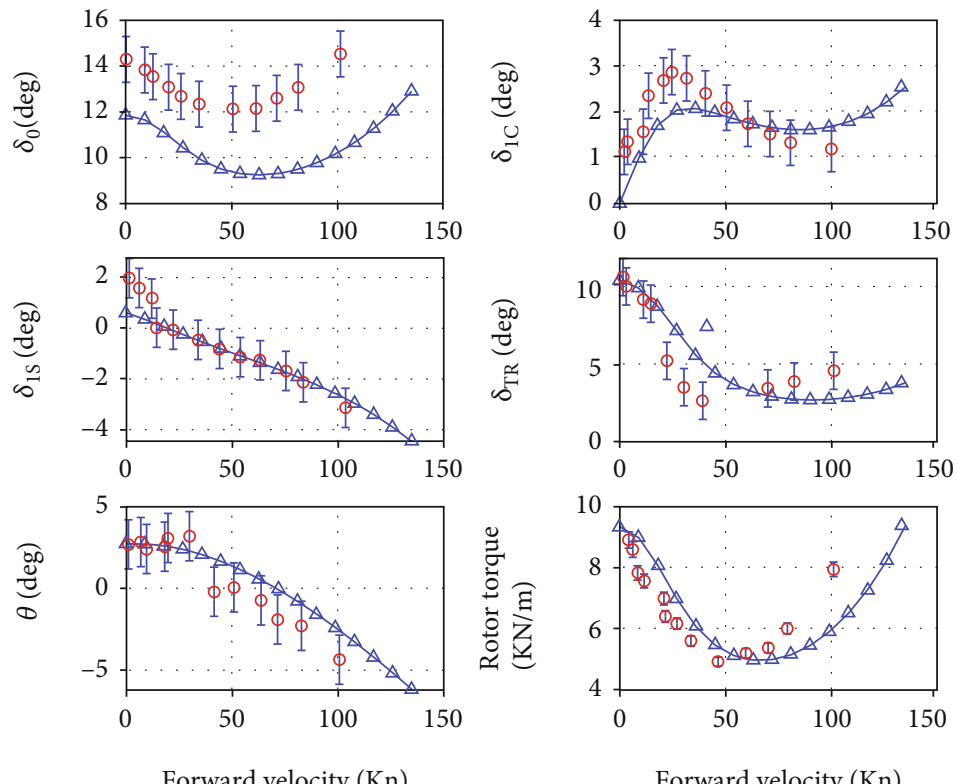

Forward velocity (Kn)

Forward velocity $(\mathrm{Kn})$

$$
\begin{array}{ll}
\triangle & \text { FDM results } \\
\bigcirc & \text { Flight test data } \\
\text { T } & \text { AC } 120 \text { Criteria }
\end{array}
$$

Figure 39: Comparisons of trim results (BO-105 flight data vs FDM).

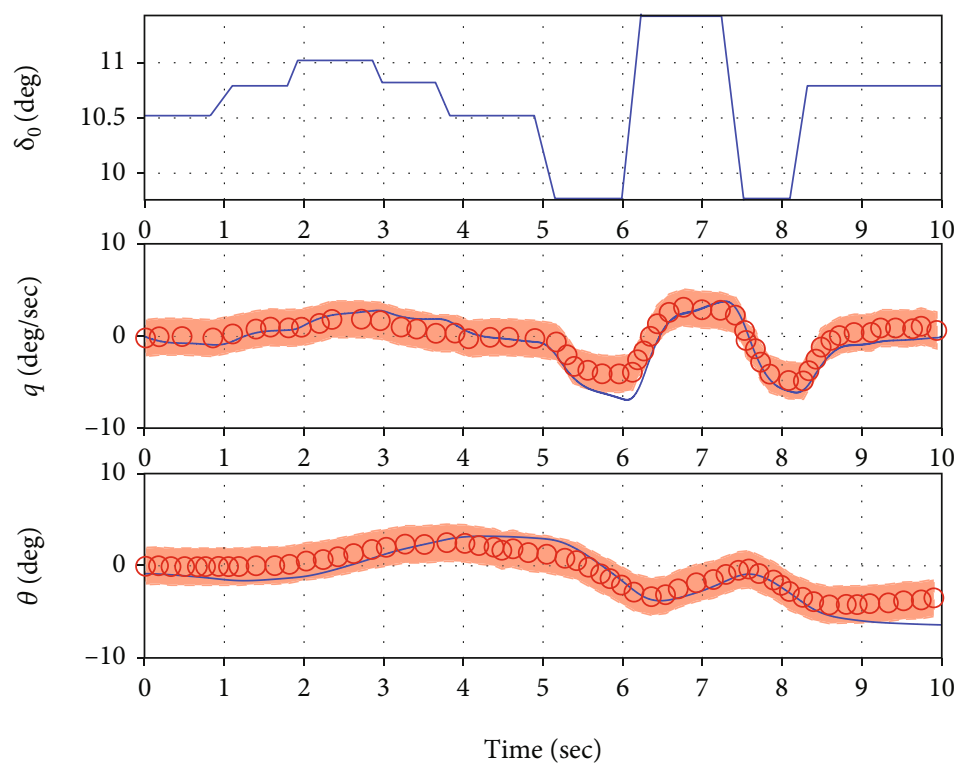

Flight test - AC 120 Criteria

_ Level 2

FIGURE 40: Comparisons of simulation results for the collective pitch response.

Figure 39 describes the comparison with the trimmed result and flight test data for forward flight. The tolerances of control input, pitch angle, and torque between flight test and analysis results are $\pm 5 \%, \pm 1.5 \%$, and $\pm 3 \%$, respectively.

Most control variables are well matched with flight test data under 40 knots flight velocity. However, the collective inputs and torque have the gap with flight test data. More- over, it is not located in the tolerance. It is judged that the phenomenon is derived by main rotor downwash effect and main rotor hub drag. The proposed FDM do not include the adjustment equations specific to individual aircraft. The accuracy of flight simulation results can be increased if the correction factors are used to lift and drag coefficients [24]. 

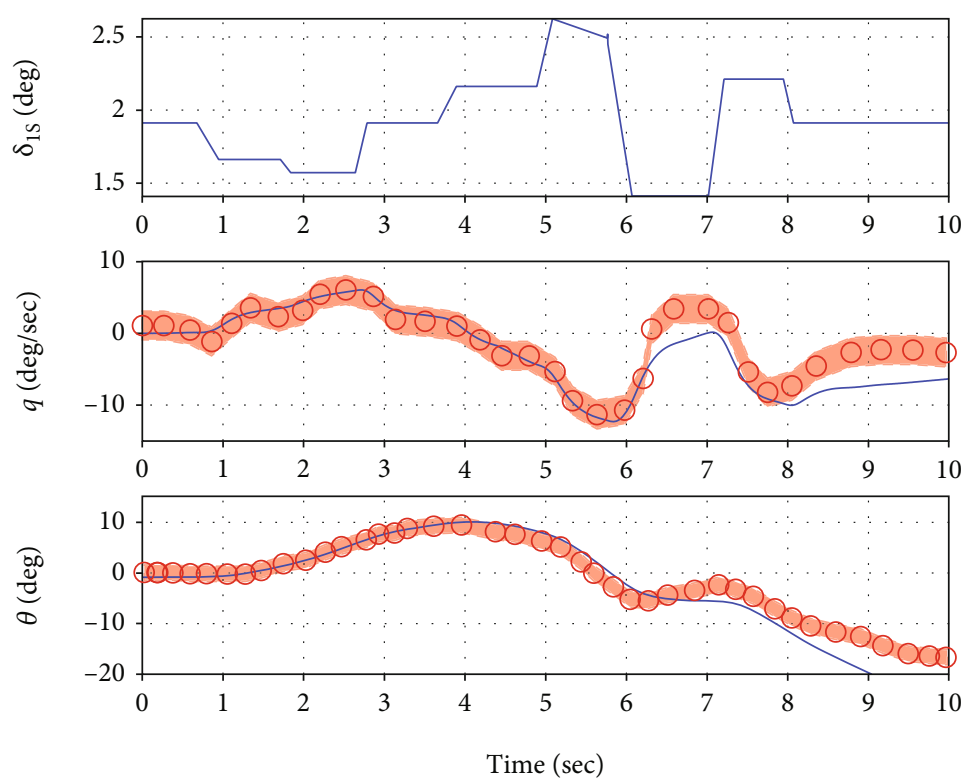

Flight test - AC 120 Criteria

- Level 2

Figure 41: Comparisons of simulation results for the cyclic response.
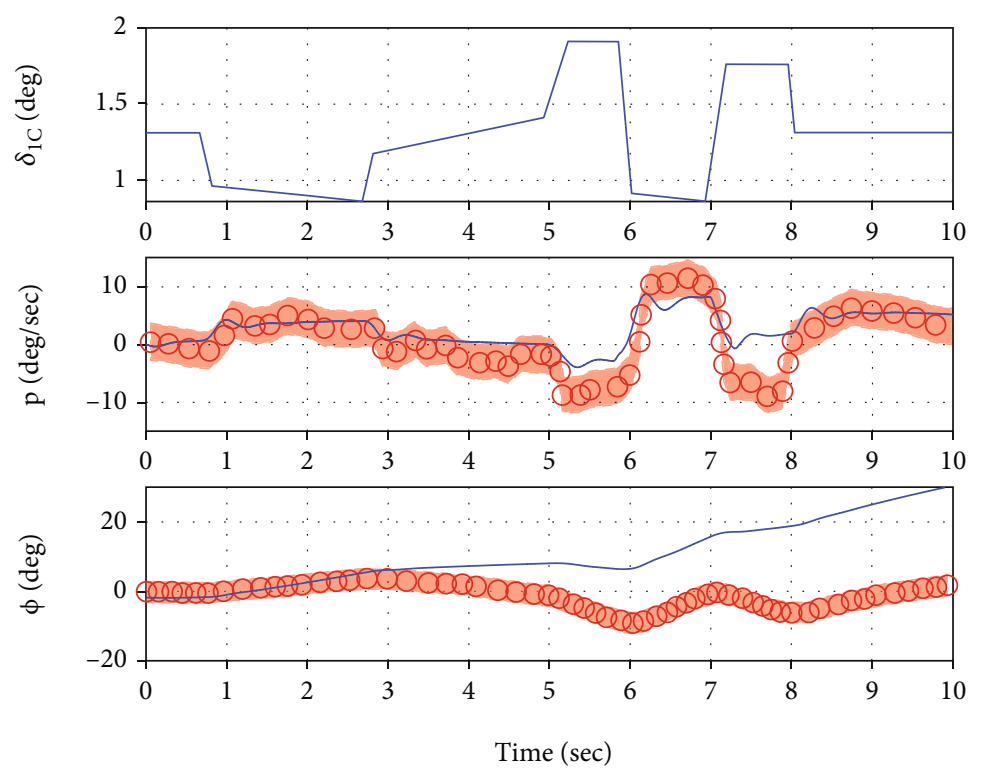

Flight test - AC 120 Criteria

— Level 2

FIGURE 42: Comparisons of simulation results for the longitudinal cyclic response.

Therefore, the aircraft performance can be accurately analyzed by proposed FDM. Furthermore, if the aircraftspecific adjustments are applied to FDM, a more precise prediction of performance can be made.

Figures 40-43 indicate the response property of aircraft for collective pitch input. The input signal is given as 3-2-1-1 for each axis. Also, it shows the handling qualities criteria of collective pitch input for longitudinal, lat- eral, and directional axis. The simulation has been conducted in forward flight with 80 knots for 10 seconds. The related responses, for example, pitch rate in longitudinal handling qualities with lateral cyclic inputs as described in Table 7 , show the very similar results with flight test data even though they have small errors. Therefore, it can be also considered that the FDM can precisely predict the flight performances. 

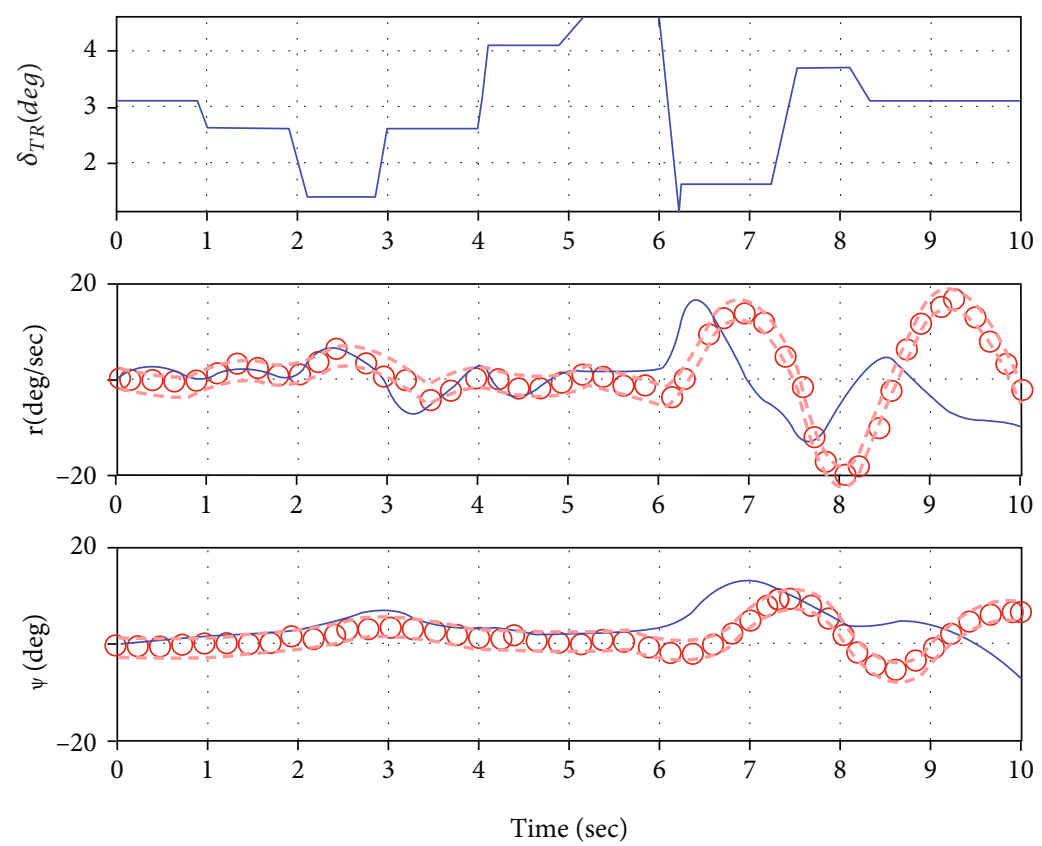

Flight test - AC 120 Criteria

Level 2

Figure 43: Comparisons of simulation results for the pedal response.

TABLE 7: AC 120-63-tolerance of trimmed flight control position and handling qualities [23].

\begin{tabular}{lcc}
\hline Test & Tolerance & Comment \\
\hline & Torque: $\pm 3.0 \%$ & Forward flight \\
Level flight performance and trimmed flight control position & Pitch attitude: $\pm 1.5^{\circ}$ & Control position: $\pm 5.0 \%$ \\
& Pitch rate: $\pm 5.0 \%$ or $\pm 2.0^{\circ} / \mathrm{sec}$ & Collective and Longitudinal \\
Longitudinal handing qualities: control response & Pitch attitude change: $\pm 10.0 \%$ or $\pm 1.5^{\circ}$ & Roll rate: $\pm 10.0 \%$ or $\pm 3.0^{\circ} / \mathrm{sec}$ \\
Lateral handing qualities: control response & Roll attitude change: $\pm 10.0 \%$ or $\pm 3.0^{\circ}$ \\
& Yaw rate: $\pm 10.0 \%$ or $\pm 2.0^{\circ} / \mathrm{sec}$ \\
Directional handing qualities: control response & Yaw attitude change: $\pm 10.0 \%$ or \pm 2.0 \\
\hline
\end{tabular}

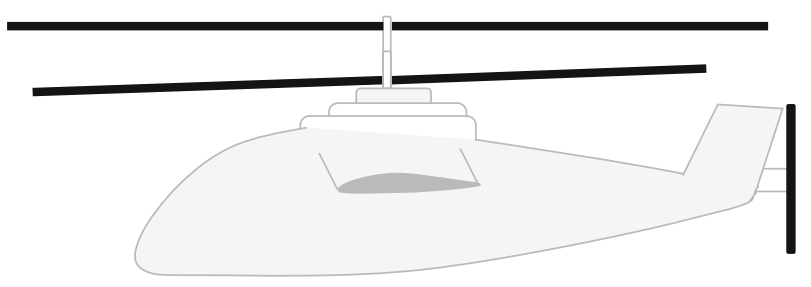

FIgURE 44: Compound rotorcraft (coaxial type).

3.4. Compound Helicopter. The flight performance analysis has been carried out for a compound rotorcraft configuration to verify the usefulness of the FDM. The configuration of compound rotorcraft has been shown in Figure 44.

The compound aircraft using coaxial rotor is based on the single rotor helicopter used in HETLAS. The coaxial rotors were assumed to be $\mathrm{ABC}$ rotors, and the blade chords were adjusted to have the same solidity. In addition, the wings were attached to the center at both sides of the fuselage, and a pusher propeller was attached to the rear fuselage. The control inputs were collective, longitudinal and lateral cyclic pitches, and differential collective pitch as given in Equation (27). Furthermore, the collective control of the pusher was applied through thrust scheduling as shown in Figure 45 to create appropriate propulsive force according to the forward flight velocity.

$$
\mathbf{u}=\left(\delta_{0}, \delta_{1 C}, \delta_{1 S}, \Delta \delta_{0}\right)^{T}
$$

Figures 46 shows the trim results of compound aircraft. The compound aircraft has a high pitch attitude angle with small roll attitude angle and lateral cyclic input due to torque from the pusher at the hovering status.

Then, the collective input is getting decreased with the pitch angle until 80 knots because of the lift of wing 


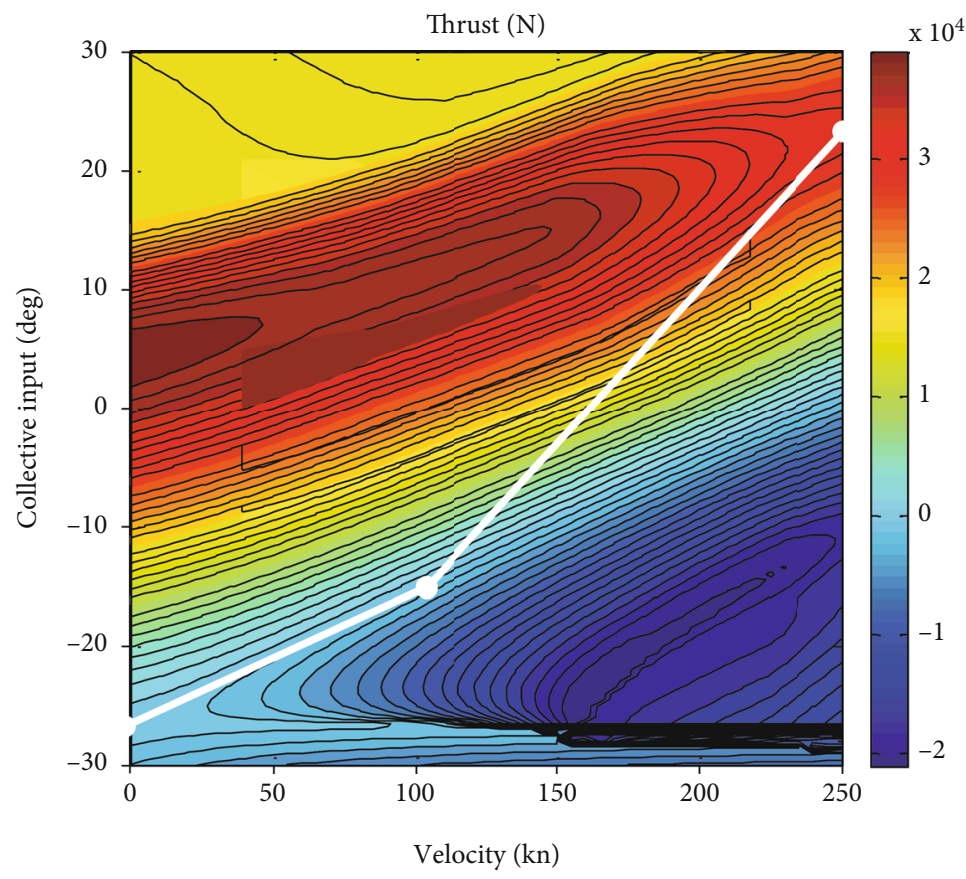

FIGURE 45: Propeller thrust scheduling with respect to forward velocity (white line).
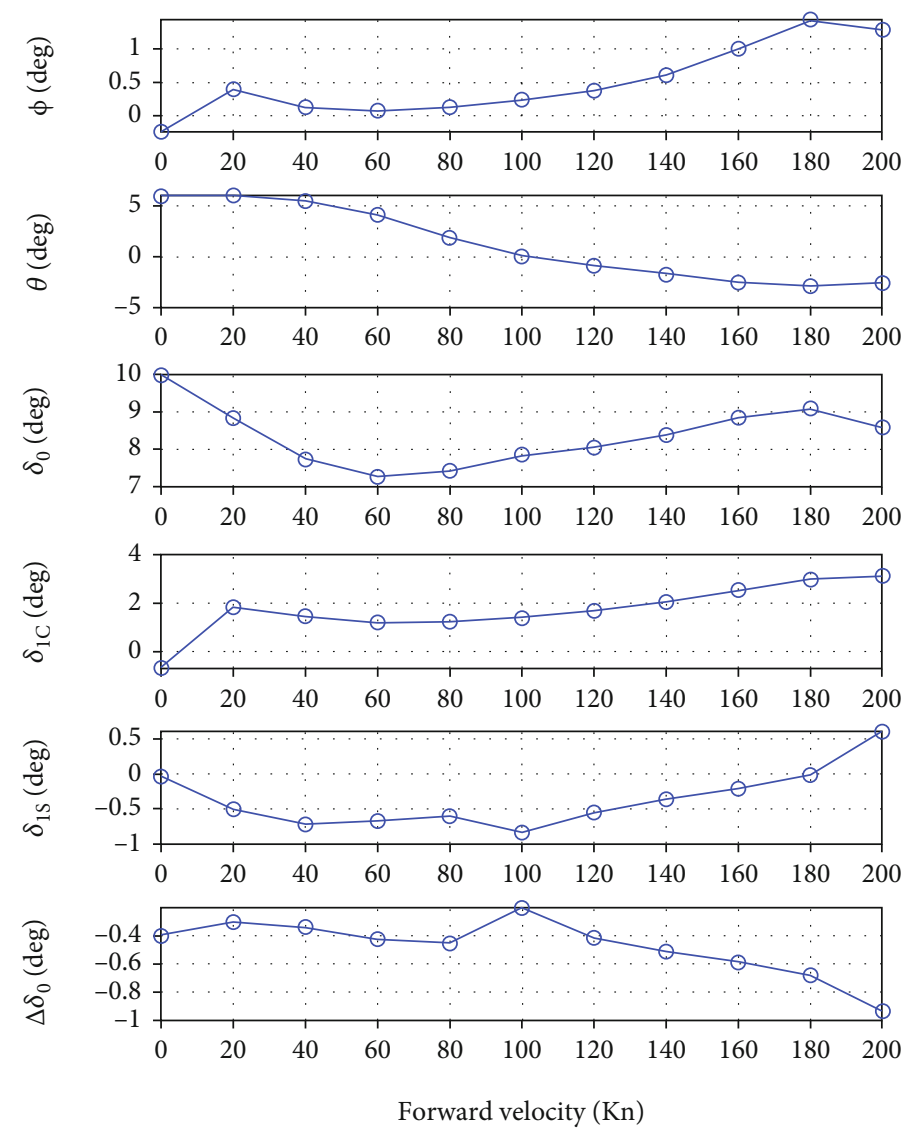

FIGURE 46: Attitude angles and control inputs for trimmed condition of the compound helicopter. 
increased. After 80 knots, the pitch angle decreased, and the pusher collective input increased more steeply. This makes lateral cyclic inputs increased due to high torque from the pusher. Although there is the complicated interplay between the longitudinal axis with collective and longitudinal cyclic input, the lateral cyclic input is working for balancing the rolling moments. Therefore, it is judged that this simulation results are physically appropriate, and the proposed FDM can be widely used to analyze the flight performance of compound aircraft.

\section{Conclusion}

This paper developed the unified high-fidelity flight dynamic modeling technique for compound aircraft. The unified rotor model calculated the aerodynamic forces and moments by using BEM with enhancement of the coefficient estimation. Furthermore, the coefficient interpolation technique has been applied to the rotor sweep, its orientation, and rotor dynamics. The proposed FDM uses the aerodynamic coefficients of airfoil for rotor and wing. The aerodynamic coefficients are the necessary data to build the high-fidelity FDM. This is the same with the traditional FDM technique. The analysis performance of unified FDM do not depend on the configuration of aircraft such as wing span and rotor diameter. Especially, although the aircraft has not manufactured, the performance analysis can be performed by unified FDM. This is the main advantage of this research, whereas the traditional FDM using experimental data has the dependency of configuration for aircraft since the experimental results is different. This is the limitation of development for compound aircraft as mentioned in section of introduction. Thus, once the aerodynamic coefficients for airfoil are given to proposed FDM, most shape of rotor and wing will be accurately analyzed.

The unified rotor model was defined as general rotor and propeller to analyze its performance as shown in Figures 32 and 33. The analysis results for rotor and propeller showed the physically appropriate rotor performance as mentioned in section of application.

The unified wing model computed its aerodynamic forces and moments using strip theory and VLM using Biot-Savart laws to estimate induced velocity. Moreover, it included the wing orientation as well as control surface influences when the induce velocity was calculated. The unified wing model was defined as the tapered wing model to check the property of lift and drag. The lift and drag coefficients calculated by the unified wing model are highly accurate. It is judged that the unified rotor and wing model proposed by this research are very precise and useful.

The unified rotor and wing models were used to build FDM. The validation of fidelity for the proposed modeling technique was performed by single rotor helicopter and compound aircraft using coaxial rotors. The applications were defined as trim and simulation with flight test data. A reference helicopter including confidential data and the BO-105 single rotor helicopter was used in section of application. First, the results of trim analysis for the reference helicopter between HETLAS and unified FDM were practically the same since the small errors were induced by enhancing the estimation accuracy of aerodynamic coefficients. Second, the correlated simulation responses with control inputs which were 3-2-1-1 control to collective, lateral cyclic, longitudinal cyclic and pedal inputs. The results of simulation computed by proposed FDM for BO-105 with the flight test data showed the simulation results of proposed FDM for BO-105 that are satisfying the qualification for helicopter simulator with small error. Third, the flight performance of compound rotorcraft using coaxial rotors was analyzed using the unified FDM as shown in Figures 45 and 46 . Figures 45 and 46 show the physically appropriate results as mention in application section.

Therefore, all type aircraft can be accurately analyzed by this research. In addition, the analysis efficiency of the compound aircraft system can be dramatically raised since the flight performance based on airworthiness standards can be interpreted by nonlinear optimal control programming (NOCP) or nonlinear programing (NLP) method. It is judged that this study can affect to develop all types of aircraft and can reduce the development cost and period by providing high-fidelity analysis results for flight performance.

\section{Data Availability}

[1] The data used to support the finding of this study are available from the first and corresponding authors upon request.

\section{Conflicts of Interest}

The authors declare that there is no conflict of interest regarding the publication of this paper.

\section{Acknowledgments}

This research was supported by the Basic Science Research Program through the National Research Foundation of Korea (NRF) funded by the Ministry of Education (No. 2020R1A6A1A03046811). Also, this work was supported by the National Research Foundation of Korea (NRF) grant funded by the Korea Government (MSIT) (NRF2020R1A2C2011955).

\section{References}

[1] K. T. Lee and K. H. Lee, "UAV - current and future," Journal of Korean Society Aeronautics and Space Science, vol. 28, no. 6, pp. 142-163, 2000.

[2] “The V/STOL Wheel," https://vertipedia.vtol.org/vstol/wheel .htm/.

[3] P. M. Basset, A. Tremolet, and T. Lefebvre, "Rotary wing UAV pre-sizing: past and present methodological approaches at Onera," Journal AerospaceLab, vol. 8, pp. 1-12, 2014.

[4] M. V. Cook, Flight dynamics principles, Elsevier, second edition edition, 2007.

[5] B. Etkins and L. D. Reid, Dynamics of Flight, John Wiley \& Sons, New York, 1996.

[6] J. S. Berndt and J. S. Bsimhttp://jsbsim.sourceforge.net/.

[7] C. J. Kim, S. H. Kim, T. Park, S. H. Park, J. W. Lee, and J. S. Ko, "Flight dynamics analyses of a propeller-driven airplane (I): 
aerodynamic and inertial modeling of the propeller," International Journal of Aeronautics and Space Science, vol. 15, no. 4, pp. 345-355, 2014.

[8] J. Leishman, "Principles of helicopter aerodynamics," in Part of Cambridge Aerospace Series, Cambridge university press, 2nd edition edition, 2006.

[9] M. S. Chaffin, "A guide to the use of the pressure disk rotor model as implemented in INS3D-UP," National Aeronautics and Space Administration, Technical Report NASA, vol. CR4692, 1995.

[10] S. Taamallah, "Flight dynamics modeling for a small-scale flybarless helicopter UAV," in AIAA Atmospheric Flight Mechanics Conference, Portland, Oregon, 2011.

[11] J. Howlett, "UH-60A black hawk engineering simulation program: volume 1: mathematical model," National Aeronautics and Space Administration, vol. CR-166309, 1981.

[12] H. A. Pearson and R. F. Anderson, "Calculation of the aerodynamic characteristics of tapered wings with partial-span flaps," Natinoal Advisory Committee for Aeronautics, vol. 665, 1939.

[13] P. D. Talbot, B. E. Tinling, W. A. Decker, and R. T. Chen, “A mathematical model of a single main rotor helicopter for piloted simulation," National Aeronautics and Space Administration, no. article TM84281, 1982.

[14] R. J. Ruddell, "Advancing blade concept (ABC) technology demonstrator," Defense Technical Information Center, USAAVRDCOM-TR-81-D-5, 1981.

[15] C. J. Kim, "Implicit formulation of rotor aeromechanic equations for helicopter flight simulation," Journal of the Korean Society for Aeronautical and Space Science, vol. 30, no. 3, pp. 8-16, 2002.

[16] D. H. Lee, Design of the Control System to Implement the Autonomous Tactical Maneuvers of the Fixed-Wing Aircraft, Konkuk University, 2019.

[17] R. Fink, USAF Stability and Control DATCOM, AFWAL-TR83-3048, 1978.

[18] Y. H. Yun, C. D. Yang, C. J. Kim, and I. J. Cho, "Flight dynamic analysis program, HETLAS for development of helicopter FBW," in Korean Society for Aeronautical \& Space Sciences 2012 Spring Conference, pp. 1270-1275, Jeju, 2012.

[19] https://ko.wikipedia.org/wiki/KAI_\%EC\%88\%98\%EB\%A6\% AC\%EC\%98\%A8/.

[20] http://www.rotorleasing.com/Specifications/BO105.html/.

[21] S. H. Lee, D. H. Lee, S. H. Hur, S. H. Kang, and C. J. Kim, “General methods of rotor and wing aerodynamics modeling for drone/UAV flight dynamic model," in Korean Society for Aeronautical \& Space Sciences 2017 Spring Conference, pp. 666-667, Samcheok, Gangwon, 2017.

[22] J. Wan, Ornicopter Multidisciplinary Analyses and Conceptual Design, Delft University of Technology, 2014.

[23] "Helicopter Simulator Qualification," in FAA Advisory Circular AC 120-63, Federal Aviation Authority, 1994.

[24] M. Kerler, J. Honle, and H.-P. Kau, Modeling of BO 105 Flight Dynamics for Research on Fuel Savings due to Single-Engine Operation, 38th European Rotorcraft Forum, Amsterdam, 2012. 